\title{
The ATLAS diboson resonance in non-supersymmetric SO(10)
}

\author{
Jason L. Evans, ${ }^{a, b}$ Natsumi Nagata, ${ }^{b}$ Keith A. Olive ${ }^{b}$ and Jiaming Zheng ${ }^{b}$ \\ a School of Physics, KIAS, \\ Seoul 130-722, Korea \\ ${ }^{b}$ William I. Fine Theoretical Physics Institute, \\ School of Physics and Astronomy, University of Minnesota, \\ Minneapolis, Minnesota 55455, U.S.A. \\ E-mail: jlevans@kias.re.kr, nagat006@umn.edu, olive@physics.umn.edu, \\ zheng@physics.umn.edu
}

ABSTRACT: $\mathrm{SO}(10)$ grand unification accommodates intermediate gauge symmetries with which gauge coupling unification can be realized without supersymmetry. In this paper, we discuss the possibility that a new massive gauge boson associated with an intermediate gauge symmetry explains the excess observed in the diboson resonance search recently reported by the ATLAS experiment. The model we find has two intermediate symmetries, $\mathrm{SU}(4)_{C} \otimes \mathrm{SU}(2)_{L} \otimes \mathrm{SU}(2)_{R}$ and $\mathrm{SU}(3)_{C} \otimes \mathrm{SU}(2)_{L} \otimes \mathrm{SU}(2)_{R} \otimes \mathrm{U}(1)_{B-L}$, where the latter gauge group is broken at the $\mathrm{TeV}$ scale. This model achieves gauge coupling unification with a unification scale sufficiently high to avoid proton decay. In addition, this model provides a good dark matter candidates, whose stability is guaranteed by a $\mathbb{Z}_{2}$ symmetry present after the spontaneous breaking of the intermediate gauge symmetries. We also discuss prospects for testing these models in the forthcoming LHC experiments and dark matter detection experiments.

KeYwords: Beyond Standard Model, GUT

ARXIV EPRINT: 1512.02184 


\section{Contents}

1 Introduction 1

2 The model 2

3 Diboson signal 10

4 Conclusion $\quad 14$

$\begin{array}{ll}\text { A Decay widths } & 15\end{array}$

\section{Introduction}

Grand unification [1] is considered to be one of the most promising frameworks for physics beyond the Standard Model (SM). SO(10) Grand Unified Theories (GUTs) [2-7] have many especially attractive features. First, the full set of SM fermions of each generation together with a right-handed neutrino is embedded into a single $\mathbf{1 6}$ chiral representation of $\mathrm{SO}(10)$. Second, anomaly cancellation in the SM can be understood naturally since $\mathrm{SO}(10)$ is free from anomalies. Third, gauge coupling unification can be realized without relying on supersymmetry, if there are intermediate-scale gauge symmetries [8-14]. Majorana mass terms of right-handed neutrinos can be generated at an intermediate scale, which may explain the smallness of neutrino masses. These interesting aspects have stimulated many extensive studies of SO(10) GUTs [15-22].

In addition, $\mathrm{SO}(10)$ GUTs with intermediate gauge symmetries predict new gauge bosons whose masses are of the order of the breaking scales of the corresponding gauge symmetries. The breaking scale of these intermediate gauge symmetries affects the running of the gauge couplings. These scales can be determined by requiring gauge coupling unification, which is highly dependent on the symmetry-breaking pattern and matter content of the model. However, we will show that it is possible to obtain a model containing $\mathrm{TeV}$-scale gauge bosons accessible at the LHC, for a particular choice of the intermediate gauge symmetries and particle content.

In this paper, we consider non-supersymmetric $\mathrm{SO}(10)$ GUT models with an intermediate gauge symmetry showing up around the $\mathrm{TeV}$ scale, and discuss the possibility that a new gauge boson associated with the intermediate gauge symmetry can explain the anomalous diboson events recently reported by the ATLAS collaboration [23]. We will discuss a model with two intermediate symmetries, $\mathrm{SU}(4)_{C} \otimes \mathrm{SU}(2)_{L} \otimes \mathrm{SU}(2)_{R}$ which is then broken to $\mathrm{SU}(3)_{C} \otimes \mathrm{SU}(2)_{L} \otimes \mathrm{SU}(2)_{R} \otimes \mathrm{U}(1)_{B-L}$. The unified and intermediate scales are determined by the renormalization group (RG) running of the gauge couplings and depend on the matter content of the theory. In the model discussed, the GUT scale is of order $10^{17} \mathrm{GeV}$ and is high enough so that the proton decay bounds can be evaded. The first intermediate scale is of order $10^{9} \mathrm{GeV}$ and is high enough that constraints from leptoquarks 
are satisfied. The second intermediate scale lies around a few $\mathrm{TeV}$ and the charged gauge bosons of $\mathrm{SU}(2)_{R}, W_{R}^{ \pm}$, can have a mass $\sim 2 \mathrm{TeV}$ and may explain the excess observed in the ATLAS diboson resonance search [23]. The possibility of this new charged gauge boson explaining the diboson anomaly is discussed in refs. [24-45]. We will see that $W_{R}^{ \pm}$in this model can also reproduce the diboson excess. Furthermore, this $W_{R}^{ \pm}$may explain another anomaly observed by the CMS collaboration in the right-handed neutrino searches with a dijet and a dilepton final state [46], as we will discuss below. Our model also predicts an additional massive boson: a neutral gauge boson $Z_{R}$. Detection of this $Z_{R}$ would be a smoking-gun signature of our model. The masses of the $Z_{R}$ can be heavier than the $W_{R}^{ \pm}$ mass, and thus evades the current LHC bound. However, it may be in reach of Run-II of the LHC, as we will discuss.

Our model also contains a promising dark matter candidate. The dark matter candidate is stabilized by a residual $\mathbb{Z}_{2}$ symmetry arising after the $\mathrm{SO}(10)$ symmetry has been completely broken to the SM gauge symmetries [47-59]. This residual $\mathbb{Z}_{2}$, as it turns out, is equivalent to matter parity [60-64]. It is tantalizing that the presence of this dark matter candidate is essential for the model to achieve gauge coupling unification. An integral part of obtaining gauge coupling unification in this set up is an intermediate scale of order a $\mathrm{TeV}$, a second intermediate scale large enough to suppress the effects of leptoquarks, and a GUT scale which is large enough to avoid proton decay. ${ }^{1}$ Given these conditions, the required matter content at low energies is severely restricted, and thus it is highly non-trivial that our model contains a good dark matter candidate. The presence of dark matter gives us another opportunity to test this model; we can probe the dark matter candidate predicted in this model in dark matter detection experiments. We also discuss this prospect in what follows. Combining dark matter search results with those obtained at the LHC experiments, we may be able to test our model in near future.

This paper is organized as follows. We first describe our model in the next section. Then, in section 3, we briefly review the ATLAS diboson anomaly, and show that the $W_{R}^{ \pm}$ in our model can explain the diboson anomaly. We also discuss the present constraints on our models from various experiments and consider the testability of this model in future LHC experiments and dark matter searches. Finally, we conclude in section 4.

\section{The model}

In this section, we describe the specific $\mathrm{SO}(10)$ model used in this analysis. This model is obtained in a similar manner to that discussed in ref. [57], except now we allow for two intermediate scales. To obtain these intermediate scales, we need to arrange some Higgs fields to acquire vacuum expectation values $(\mathrm{VEVs})$ in specific directions at particular scales. Throughout this work, we make an assumption on the Higgs field content. We assume that at each intermediate scale the Higgs fields present are components of the $\mathrm{SO}(10)$ multiplets that have VEVs equal to or less then the corresponding intermediate scale. The other components have masses of order of the previous symmetry-breaking scale. We also allow for a light $\mathrm{SO}(10)$ component which could be dark matter.

\footnotetext{
${ }^{1}$ Realization of a few $\mathrm{TeV}$ intermediate scale within the framework of grand unification is also discussed in refs. [65-67].
} 
Before we get into the details of our model, we first discuss the necessity for two intermediate scales. If there were only one intermediate scale and we wished to explain the ATLAS diboson excess, we would have to choose the TeV scale. For intermediate gauge symmetries which include the $\mathrm{SU}(4)_{C}$ subgroup, this choice turns out to be problematic since the breaking of the $\mathrm{SU}(4)_{C}$ subgroup generates a vector leptoquark at that intermediate scale. Since this leptoquark interacts with both left- and right-handed fermions, it will generate excessively large contributions to the meson decays (see, e.g., ref. [68]). In fact, one can show that no tuning can simultaneously tune away the contribution of a vector leptoquark to the Kaon decays $K^{+} \rightarrow \pi^{+} \bar{e} \mu$ and $K_{L} \rightarrow \bar{e} \mu$ by exploiting the contributions of the scalar leptoquarks contained inside the Higgs fields. ${ }^{2}$ If the intermediate gauge symmetries do not contain the $\mathrm{SU}(4)_{C}$ subgroup, on the other hand, we find that we cannot obtain gauge coupling unification with a sufficiently high GUT scale since there are no new contributions to the running of the $\mathrm{SU}(3)_{C}$ gauge coupling constant. This makes it impossible to get the intermediate scale to be around the $\mathrm{TeV}$ scale while maintaining gauge coupling unification with a sufficiently high GUT scale. These problems, however, can be evaded if a two step breaking pattern is considered. The vector leptoquark then has a mass of order of the larger intermediate scale. This larger intermediate scale generates a mass for the vector leptoquarks which is high enough to avoid constraints coming from meson decays but low enough that the vector leptoquarks still contribute significantly to the running of the $\mathrm{SU}(3)_{C}$ gauge coupling.

The breaking pattern of our model, with two intermediate gauge symmetries, is from $\mathrm{SO}(10)$ to $\mathrm{SU}(4)_{C} \otimes \mathrm{SU}(2)_{L} \otimes \mathrm{SU}(2)_{R}$ which is then broken to $\mathrm{SU}(3)_{C} \otimes \mathrm{SU}(2)_{L} \otimes \mathrm{SU}(2)_{R} \otimes$ $\mathrm{U}(1)_{B-L}$. For the breaking pattern we consider, $\mathrm{SO}(10)$ is broken by the VEV of the $(\mathbf{1}, \mathbf{1}, \mathbf{1})$ component of a $\mathbf{2 1 0}$ into $\mathrm{SU}(4)_{C} \otimes \mathrm{SU}(2)_{L} \otimes \mathrm{SU}(2)_{R}$. This symmetry is then subsequently broken by a VEV of the $(\mathbf{1 5}, \mathbf{1}, \mathbf{1})$ component of the same $\mathbf{2 1 0}$ to $\mathrm{SU}(3)_{C} \otimes$ $\mathrm{SU}(2)_{L} \otimes \mathrm{SU}(2)_{R} \otimes \mathrm{U}(1)_{B-L} . \quad$ Importantly, the $(\mathbf{1}, \mathbf{1}, \mathbf{1})$ component of the 210 breaks $D$-parity [69-73] at the GUT scale, and thus the low-energy effective theory does not exhibit a left-right symmetry [74-77]. Finally, this gauge group is broken to the SM gauge symmetries at the $\mathrm{TeV}$ scale by the $(\mathbf{1}, \mathbf{1}, \mathbf{3},+2)$ component of a $\mathbf{1 2 6}$. However, there remains a $\mathbb{Z}_{2}$ symmetry coming from $\mathrm{U}(1)_{B-L}$ since the $B-L$ charge of this component of the $\mathbf{1 2 6}$ is $2[47-51]$. The $(\mathbf{1}, \mathbf{1}, \mathbf{3}, 0)$ component of the $\mathbf{2 1 0}$ also breaks $\mathrm{SU}(3)_{C} \otimes \mathrm{SU}(2)_{L} \otimes$ $\mathrm{SU}(2)_{R} \otimes \mathrm{U}(1)_{B-L}$, but does not break the $\mathbb{Z}_{2}$ symmetry since its $B-L$ charge is zero. In summary, we consider the following symmetry-breaking chain:

$$
\begin{aligned}
\mathrm{SO}(10) \stackrel{\langle\mathbf{2 1 0}\rangle}{\longrightarrow} \mathrm{SU}(4)_{C} \otimes \mathrm{SU}(2)_{L} \otimes \mathrm{SU}(2)_{R} \\
\stackrel{\langle\mathbf{2 1 0}\rangle}{\longrightarrow} \mathrm{SU}(3)_{C} \otimes \mathrm{SU}(2)_{L} \otimes \mathrm{SU}(2)_{R} \otimes \mathrm{U}(1)_{B-L} \\
\stackrel{\langle\mathbf{1 2 6}\rangle\langle\mathbf{2 1 0}\rangle}{\longrightarrow} \mathrm{SU}(3)_{C} \otimes \mathrm{SU}(2)_{L} \otimes \mathrm{U}(1)_{Y} \otimes \mathbb{Z}_{2} .
\end{aligned}
$$

\footnotetext{
${ }^{2}$ This is because both of these decays arise from the same effective operator. These two decays depend on the vector current and axial current respectively. Because the effective operators generated from scalar leptoquarks will have different chirality for the quarks than the vector leptoquark, the contribution from the scalar leptoquark will add to one decay and subtract from the others. Therefore, no tuning can remove this contribution.
} 
Under the remnant $\mathbb{Z}_{2}$ symmetry, which is found to be equivalent to matter parity $P_{M}=$ $(-1)^{3(B-L)}$ [60-64], the SM fermions are odd while the SM Higgs is even. Therefore, a scalar boson (fermion) can be stable if it is odd (even) under this $\mathbb{Z}_{2}$ symmetry. If such a particle is electrically and color neutral, it can be a good dark matter candidate [52-55]. For a detailed discussions on this class of dark matter candidates in $\mathrm{SO}(10)$ GUTs, see refs. $[56,57]$.

The low-energy matter content of this model, beyond the SM fermions and righthanded neutrinos (which are embedded into three 16 representations), is given in table 1. Here, the first, second, and third columns show the $\mathrm{SO}(10), \mathrm{SU}(4)_{C} \otimes \mathrm{SU}(2)_{L} \otimes \mathrm{SU}(2)_{R}$, and $\mathrm{SU}(3)_{C} \otimes \mathrm{SU}(2)_{L} \otimes \mathrm{SU}(2)_{R} \otimes \mathrm{U}(1)_{B-L}$ quantum numbers of the particles, respectively, while the fourth column represents their $\mathbb{Z}_{2}$ charges. The subscripts $C$ and $D$ refer to complex and Dirac respectively. Most of the components of the $\mathbf{2 1 0}$ have GUT scale masses and do not affect our analysis. In this model, the $(\mathbf{1 5}, \mathbf{1}, \mathbf{1})_{C}$ component of $\mathbf{2 1 0}_{C}$ has a mass similar to the $\mathrm{SU}(4)_{C} \otimes \mathrm{SU}(2)_{L} \otimes \mathrm{SU}(2)_{R}$ breaking scale, as its VEV breaks the symmetry. Similarly, the components of $(\mathbf{1 5}, \mathbf{1}, \mathbf{3}) \in \mathbf{2 1 0}$ and $(\overline{\mathbf{1 0}}, \mathbf{1}, \mathbf{3}) \in \mathbf{1 2 6}$ charged under color have masses of order the higher intermediate scale breaking. The components in the third column of the table are those components which are fine-tuned to have masses lighter than this scale. Among the light fields are the $(\mathbf{1}, \mathbf{1}, \mathbf{3},+2)_{C}$ and the $(\mathbf{1}, \mathbf{1}, \mathbf{3}, 0)_{C}$ which obtain VEVs and break the $\mathrm{SU}(3)_{C} \otimes \mathrm{SU}(2)_{L} \otimes \mathrm{SU}(2)_{R} \otimes \mathrm{U}(1)_{B-L}$ symmetry to the SM symmetries. The components of these fields which are not eaten by the gauge bosons receive masses of order of the VEV breaking this symmetry. The rest of the Higgs fields listed in the table are responsible for breaking the electroweak symmetry and have masses of order the electroweak scale. Also listed in the table is an $\mathrm{SU}(2)_{L}$ triplet Dirac fermion which can be a viable dark matter candidate and could originate from a $\mathbf{4 5}$ of $\mathrm{SO}(10)$. The remaining components of the $\mathbf{4 5}$ (beyond the triplet) should all have GUT scale masses. In summary, the states in column 2 affect the running of the gauge couplings between the high intermediate scale and the GUT scale, and those in column 3 affect the running between the TeV scale and the high intermediate scale. The particles in the third column and green shaded rows contribute to the running of the gauge couplings below the TeV scale. This leads to a theory below the TeV scale with the SM fermions and four doublet Higgs bosons. ${ }^{3}$ A similar decomposition can be made for the the gauge bosons; the $(\mathbf{6}, \mathbf{2}, \mathbf{2})$ component of the $\mathrm{SO}(10)$ gauge boson, $\mathbf{4 5}$, is around the GUT scale, while the other components, the $\mathrm{SU}(4)_{C}, \mathrm{SU}(2)_{L}$, and $\mathrm{SU}(2)_{R}$ gauge bosons, are massless at this stage of the GUT symmetry breaking. A part of the $\mathrm{SU}(4)_{C}$ gauge bosons, the vector leptoquark, obtains a mass of the order of the higher intermediate scale, as we will see below.

The mass of the dark matter candidate is fixed by requiring that its thermal relic abundance agree with the observed dark matter density $\Omega_{\mathrm{DM}} h^{2} \simeq 0.12$ [78]. The thermal relic abundance of any DM candidate is determined by its thermally averaged annihilation cross section $\sigma_{\text {ann }}$ times the relative velocity between the initial particles, $v_{\text {rel }}$, via the following relation:

$$
\Omega_{\mathrm{DM}} h^{2} \simeq \frac{3 \times 10^{-27} \mathrm{~cm}^{3} \mathrm{~s}^{-1}}{\left\langle\sigma_{\mathrm{ann}} v_{\mathrm{rel}}\right\rangle} .
$$

\footnotetext{
${ }^{3}$ As we will see below, the dark matter candidate will need to be of order the TeV scale.
} 


\begin{tabular}{|cccc|}
\hline $\mathrm{SO}(10)$ & $\mathrm{SU}(4)_{C} \otimes \mathrm{SU}(2)_{L} \otimes \mathrm{SU}(2)_{R}$ & $\mathrm{SU}(3)_{C} \otimes \mathrm{SU}(2)_{L} \otimes \mathrm{SU}(2)_{R} \otimes \mathrm{U}(1)_{B-L}$ & $\mathbb{Z}_{2}$ \\
\hline $\mathbf{2 1 0}_{C}$ & $(\mathbf{1 5}, \mathbf{1}, \mathbf{1})_{C}$ & - & + \\
$\mathbf{1 2 6}_{C}$ & $(\overline{\mathbf{1 0}}, \mathbf{1}, \mathbf{3})_{C}$ & $(\mathbf{1}, \mathbf{1}, \mathbf{3},+2)_{C}$ & + \\
$\mathbf{2 1 0}_{C}$ & $(\mathbf{1 5}, \mathbf{1}, \mathbf{3})_{C}$ & $(\mathbf{1}, \mathbf{1}, \mathbf{3}, 0)_{C}$ & + \\
$\mathbf{1 0}_{C}$ & $(\mathbf{1}, \mathbf{2}, \mathbf{2})_{C}$ & $(\mathbf{1}, \mathbf{2}, \mathbf{2}, 0)_{C}$ & + \\
$\mathbf{1 2 6}_{C}$ & $(\mathbf{1 5}, \mathbf{2}, \mathbf{2})_{C}$ & $(\mathbf{1}, \mathbf{2}, \mathbf{2}, 0)_{C}$ & + \\
$\mathbf{4 5}_{D}$ & $(\mathbf{1}, \mathbf{3}, \mathbf{1})_{D}$ & $(\mathbf{1}, \mathbf{3}, \mathbf{1}, 0)_{D}$ & + \\
\hline
\end{tabular}

Table 1. Particle content of our model. The first, second, and third columns show the $\mathrm{SO}(10)$, $\mathrm{SU}(4)_{C} \otimes \mathrm{SU}(2)_{L} \otimes \mathrm{SU}(2)_{R}$, and $\mathrm{SU}(3)_{C} \otimes \mathrm{SU}(2)_{L} \otimes \mathrm{SU}(2)_{R} \otimes \mathrm{U}(1)_{B-L}$ quantum numbers of the particles, respectively, while the forth column represents their $\mathbb{Z}_{2}$ charges. The orange (green) shaded fields acquire VEVs of about a few $\mathrm{TeV}$ (the electroweak scale), and the blue shaded particle contains a dark matter candidate. The $(\mathbf{1 5}, \mathbf{1}, \mathbf{1})_{C}$ of the $\mathbf{2 1 0}_{C}$ lies around the $\mathrm{SU}(4)_{C} \otimes \mathrm{SU}(2)_{L} \otimes$ $\mathrm{SU}(2)_{R}$ breaking scale, and none of its components appears below this scale.

Here, the brackets signify a thermal average. In the case of an $\mathrm{SU}(2)_{L}$ triplet Dirac fermion dark matter candidate with zero hypercharge, the $s$-wave annihilation cross section is given by $[79-81]$

$$
\left\langle\sigma_{\text {ann }} v_{\text {rel }}\right\rangle \simeq \frac{37 g_{2}^{4}}{192 \pi m_{\mathrm{DM}}^{2}},
$$

where $g_{2}$ denotes the $\mathrm{SU}(2)_{L}$ gauge coupling constant and $m_{\mathrm{DM}}$ is the dark matter mass. From eqs. (2.2) and (2.3), we obtain $m_{\mathrm{DM}} \simeq 2 \mathrm{TeV}$. However, it is known that the nonperturbative Sommerfeld effect significantly enhances the annihilation cross section [82, 83], which results in a smaller cross section, and thus larger dark matter mass, needed to reproduce the correct dark matter density. As far as we know, the thermal relic abundance of a triplet Dirac fermion has not been computed yet with the Sommerfeld enhancement effect included. However, we expect that the favored mass for a Dirac triplet is smaller than that for a Majorana triplet (which was found to be $\sim 2.7 \mathrm{TeV}[84]$ ), since the thermallyaveraged annihilation cross section of Dirac dark matter is a factor of two smaller than that of Majorana dark matter.

In figure 1, we show a plot of the running of the gauge coupling constants in this model. Here, we set the masses of the fields in the third column in table 1 to be $1.9 \mathrm{TeV}$. In this figure, we show the running of the inverse of $\alpha_{a} \equiv g_{a}^{2} /(4 \pi)(a=1,2,3)$ with $g_{1}$, $g_{2}$, and $g_{3}$ corresponding to the $\mathrm{U}(1)_{Y}, \mathrm{SU}(2)_{L}$, and $\mathrm{SU}(3)_{C}$ gauge coupling constants, respectively. The definitions of these gauge couplings in the $\mathrm{SU}(4)_{C} \otimes \mathrm{SU}(2)_{L} \otimes \mathrm{SU}(2)_{R}$ theory are given by

$$
\frac{1}{\alpha_{1}} \equiv \frac{3}{5} \frac{1}{\alpha_{2 R}}+\frac{2}{5} \frac{1}{\alpha_{4}}, \quad \alpha_{2} \equiv \alpha_{2 L}, \quad \alpha_{3} \equiv \alpha_{4},
$$

where $\alpha_{4}, \alpha_{2 L}$, and $\alpha_{2 R}$ represent the $\mathrm{SU}(4)_{C}, \mathrm{SU}(2)_{L}$, and $\mathrm{SU}(2)_{R}$ gauge couplings, respectively, while those in the $\mathrm{SU}(3)_{C} \otimes \mathrm{SU}(2)_{L} \otimes \mathrm{SU}(2)_{R} \otimes \mathrm{U}(1)_{B-L}$ theory are

$$
\frac{1}{\alpha_{1}} \equiv \frac{3}{5} \frac{1}{\alpha_{2 R}}+\frac{2}{5} \frac{1}{\alpha_{B-L}}, \quad \alpha_{2} \equiv \alpha_{2 L}
$$




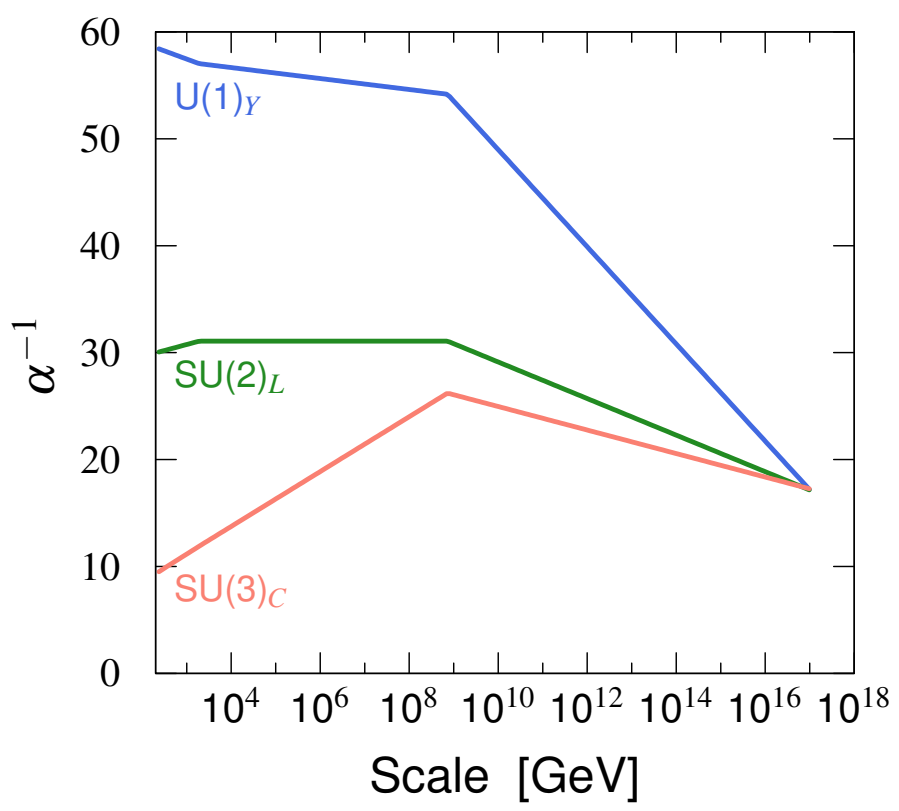

Figure 1. Running of gauge couplings in our model. Here, we set the masses of the fields in the third column in table 1 to be $1.9 \mathrm{TeV}$.

where $\alpha_{B-L}$ is the $\mathrm{U}(1)_{B-L}$ gauge coupling. Using the weak scale values of the gauge couplings as inputs and insisting on unification of the coupling constants at some high energy scale, allows us to use the three RGEs to solve for the higher intermediate scale, the GUT scale, and the value of the the unified gauge coupling at the GUT scale (recall that we have fixed the value of the lower intermediate scale). From this figure, we find that the GUT scale in this model is $9.8 \times 10^{16} \mathrm{GeV}$, which is high enough to avoid proton decay constraints. The $\mathrm{SU}(4)_{C} \otimes \mathrm{SU}(2)_{L} \otimes \mathrm{SU}(2)_{R}$ breaking scale is determined to be $\simeq 7.2 \times 10^{8} \mathrm{GeV}$. The $\mathrm{SU}(3)_{C} \otimes \mathrm{SU}(2)_{L} \otimes \mathrm{SU}(2)_{R} \otimes \mathrm{U}(1)_{B-L}$ gauge coupling constants at $1.9 \mathrm{TeV}$ are found to be

$$
g_{B-L} \simeq 0.58, \quad g_{2 L} \simeq 0.64, \quad g_{2 R} \simeq 0.42, \quad g_{3} \simeq 1.03 .
$$

The value of $g_{2 R}$ above will be important when we discuss the diboson anomaly.

In this model, only $W_{R}^{ \pm}$and $Z_{R}$ lie around the TeV scale, while the vector leptoquarks have masses of $\mathcal{O}\left(10^{8-9}\right) \mathrm{GeV}$. The scalar leptoquarks arising from the $(\mathbf{1 5}, \mathbf{1}, \mathbf{1})_{C}$ and $(\mathbf{1 5}, \mathbf{1}, \mathbf{3})_{C}$ of the $\mathbf{2 1 0}_{C}$ and $(\overline{\mathbf{1 0}}, \mathbf{1}, \mathbf{3})_{C}$ and $(\mathbf{1 5}, \mathbf{2}, \mathbf{2})_{C}$ of the $\mathbf{1 2 6}_{C}$ also have a mass of order this scale. The masses of $W_{R}^{ \pm}$and $Z_{R}$ are computed to be

$$
\begin{aligned}
& m_{W_{R}}^{2} \simeq g_{2 R}^{2}\left[\left(v_{R}^{\mathbf{1 0}}\right)^{2}+\left(v_{R}^{\mathbf{1 5}}\right)^{2}\right], \\
& m_{Z_{R}}^{2} \simeq 2\left(g_{2 R}^{2}+\frac{3}{2} g_{B-L}^{2}\right)\left(v_{R}^{\mathbf{1 0}}\right)^{2},
\end{aligned}
$$

where

$$
\left\langle(\mathbf{1}, \mathbf{1}, \mathbf{3},+2)_{C}\right\rangle=T_{-} v_{R}^{\mathbf{1 0}}, \quad\left\langle(\mathbf{1}, \mathbf{1}, \mathbf{3}, 0)_{C}\right\rangle=T_{3} v_{R}^{\mathbf{1 5}} .
$$

Here, $T_{a}=\tau_{a} / 2(a=1,2,3)$ with $\tau_{a}$ the Pauli matrices and $T_{-} \equiv T_{1}-i T_{2}$, and we have neglected the contribution of the electroweak-scale VEVs for simplicity. On the other 
hand, the vector leptoquarks, whose quantum numbers under the $\mathrm{SU}(3)_{C} \otimes \mathrm{SU}(2)_{L} \otimes \mathrm{U}(1)_{Y}$ symmetry are $(\mathbf{3}, \mathbf{1},+2 / 3) \oplus(\overline{\mathbf{3}}, \mathbf{1},-2 / 3)$, acquire a mass of

$$
m_{V_{\mathrm{LQ}}}^{2} \simeq \frac{2}{3} g_{4}^{2}\left(v_{422}^{\mathbf{1 5}}\right)^{2}
$$

with

$$
\left\langle(\mathbf{1 5}, \mathbf{1}, \mathbf{1})_{C}\right\rangle=X_{B-L} v_{422}^{\mathbf{1 5}},
$$

where $X_{B-L}$ is the $\mathrm{SU}(4)_{C}$ generator corresponding to the $B-L$ charge, i.e. $\sqrt{\frac{3}{8}}(B-L) .{ }^{4}$ As the $(\mathbf{1 5}, \mathbf{1}, \mathbf{1})_{C} \mathrm{VEV} v_{422}^{\mathbf{1 5}}$ sets the $\mathrm{SU}(4)_{C} \otimes \mathrm{SU}(2)_{L} \otimes \mathrm{SU}(2)_{L}$ breaking scale, $m_{V_{\mathrm{LQ}}}$ is as high as $\sim 10^{9} \mathrm{GeV}$. The masses of the scalar leptoquarks are dependent on the couplings in the scalar potential; generically, they are also $\mathcal{O}\left(v_{422}^{\mathbf{1 5}}\right)$.

Since the gauge couplings $g_{B-L}$ and $g_{2 R}$ are determined, and given in eq. (2.6), if we fix the $W_{R}^{ \pm}$mass, then we obtain the $Z_{R}$ mass as a function of $v_{R}^{\mathbf{1 5}}$. We show this in figure 2 setting $m_{W_{R}}=1.9 \mathrm{TeV}$. It is found that the $Z_{R}$ mass is relatively small; in particular, $m_{Z_{R}}$ can be much smaller than that predicted in the minimal left-right symmetric model with a single $\mathrm{SU}(2)_{R}$ triplet Higgs field [74-77], which is reproduced if one takes $v_{R}^{\mathbf{1 5}}=0$. This distinguishing feature can be tested at the LHC. Currently, the most stringent bound on $m_{Z_{R}}$ is given by the resonance searches in Drell-Yan processes [85, 86]. The CMS collaboration gives the most severe limits on this process [86]. Using this analysis, ${ }^{5}$ it is found that the present lower bound on the $Z_{R}$ mass in our model is $m_{Z_{R}} \gtrsim 3.05 \mathrm{TeV}{ }^{6}$ We show this limit by the blue shaded area in figure 2 . We find that $m_{Z_{R}}$ in our model can satisfy this constraint over a wide range of $v_{R}^{\mathbf{1 5}}$. The rest of allowed mass range, $3.05 \mathrm{TeV} \lesssim m_{Z_{R}} \lesssim 5.2 \mathrm{TeV}$, can be reached at Run-II of the LHC. For example, the $14 \mathrm{TeV}$ LHC run with an integrated luminosity of $300 \mathrm{fb}^{-1}$ can probe the entire parameter

\footnotetext{
${ }^{4}$ As a simple cross check, we here note that for a generic gauge theory which is spontaneously broken by a VEV of a Higgs field $\langle\varphi\rangle$, the trace of the mass matrix $M_{A B}^{2}$ of the gauge bosons is given by

$$
\sum_{A} M_{A A}^{2}=2 g_{G}^{2}\langle\varphi\rangle^{*} T_{A} T_{A}\langle\varphi\rangle=2 g_{G}^{2} C_{G}(R)|\langle\varphi\rangle|^{2},
$$

where $C_{G}(R)$ denotes the quadratic Casimir invariant for a representation $R$ of a gauge group $G$. For example, the vector leptoquark mass $m_{V_{\mathrm{LQ}}}^{2}$ is derived from the relation as

$$
6 m_{V_{\mathrm{LQ}}}^{2}=2 g_{4}^{2} C_{\mathrm{SU}(4)}(\mathbf{1 5}) \cdot\left|\left\langle(\mathbf{1 5}, \mathbf{1}, \mathbf{1})_{C}\right\rangle\right|^{2} .
$$

For the TeV-scale gauge bosons, on the other hand, we have

$$
\begin{aligned}
2 m_{W_{R}}^{2}+m_{Z_{R}}^{2}= & 2 g_{2 R}^{2} C_{\mathrm{SU}(2)}(\mathbf{3}) \cdot\left[\left|\left\langle(\mathbf{1}, \mathbf{1}, \mathbf{3},+2)_{C}\right\rangle\right|^{2}+\left|\left\langle(\mathbf{1}, \mathbf{1}, \mathbf{3}, 0)_{C}\right\rangle\right|^{2}\right] \\
& +2 g_{B-L}^{2}\left(2 \sqrt{\frac{3}{8}}\right)^{2} \cdot\left|\left\langle(\mathbf{1}, \mathbf{1}, \mathbf{3},+2)_{C}\right\rangle\right|^{2}
\end{aligned}
$$

These two relations are consistent with eqs. (2.7) and (2.9).

${ }^{5}$ The CMS limits are given in terms of the two parameters $c_{u}$ and $c_{d}$, which are defined in [87], as $c_{u, d}=\left(g^{\prime 2} / 2\right)\left(g_{V}^{u, d^{2}}+g_{A}^{u, d^{2}}\right) \operatorname{Br}(\ell \ell)$, where $\operatorname{Br}(\ell \ell)$ is the branching fraction into lepton pairs, and $g^{\prime} g_{V}^{u(d)}$ and $g^{\prime} g_{A}^{u(d)}$ are the vector and axial-vector couplings of $Z_{R}$ with up-type (down-type) quarks, respectively. Using these expressions we find that our model gives $c_{u} \simeq 2 \times 10^{-3}$ and $c_{d} \simeq 1 \times 10^{-2}$, which leads to our stated mass bound on $Z_{R}$.

${ }^{6}$ This limit is relaxed slightly if one allows $Z_{R}$ to decay into right-handed neutrinos.
} 


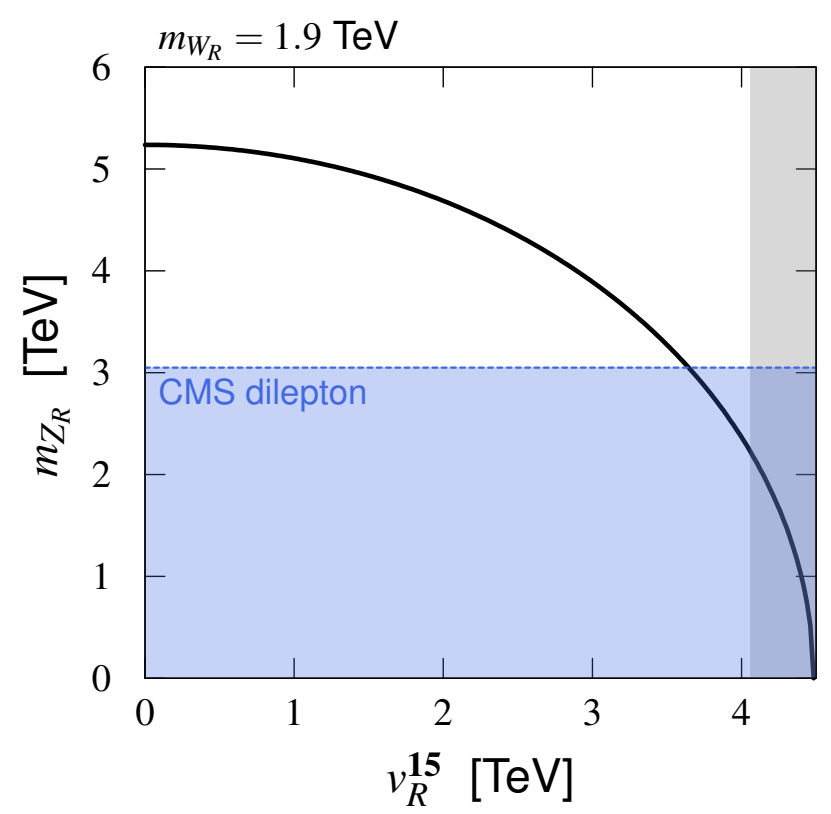

Figure 2. $m_{Z_{R}}$ as a function of $v_{R}^{\mathbf{1 5}}$. Here, we set $m_{W_{R}}=1.9 \mathrm{TeV}$. The gray shaded region predicts right-handed neutrino masses to be lighter than the $W_{R}^{ \pm}$mass when $y_{\Delta}<1$, while the blue shaded area is excluded by the CMS dilepton searches [86].

space in figure 2 [88]. Finally, we note in passing that the $Z_{R}$ in our model may explain the dielectron event at $2.9 \mathrm{TeV}$ recently announced by the CMS collaboration [89], although there are inconsistencies with the CMS dilepton bound. These $Z_{R}$ signatures can possibly be tested in the near future.

The VEVs of the bi-doublet Higgs fields $\Phi_{\mathbf{1}}$ and $\Phi_{\mathbf{1 5}}$ originally coming from $(\mathbf{1}, \mathbf{2}, \mathbf{2})_{C}$ and $(\mathbf{1 5}, \mathbf{2}, \mathbf{2})_{C}$ of the $\mathbf{1 0}_{C}$ and $\mathbf{1 2 6}_{C}$, respectively, induce mixing between the $\mathrm{SU}(2)_{L}$ and $\mathrm{SU}(2)_{R}$ gauge bosons. Through this mixing, $W_{R}^{ \pm}$can decay into a $W$ and $Z$. Therefore, by appropriately choosing the mixing angle, we may explain the diboson excess observed in the ATLAS experiment [23], as we discuss in the next section.

In addition, the VEVs of $\Phi_{\mathbf{1}}, \Phi_{\mathbf{1 5}}$, and $\Delta=(\mathbf{1}, \mathbf{1}, \mathbf{3},+2)_{C}$ from the $\mathbf{1 2 6}_{C}$ generate the fermion mass terms via Yukawa interactions. At the $\mathrm{TeV}$ scale, the relevant Yukawa terms are given by

$$
\begin{aligned}
\mathcal{L}_{\text {Yukawa }}= & -\overline{Q_{L}}\left(y_{1}^{Q} \Phi_{\mathbf{1}}+\widetilde{y}_{1}^{Q} \widetilde{\Phi}_{\mathbf{1}}\right) Q_{R}-\frac{1}{\sqrt{24}} \overline{Q_{L}}\left(y_{15}^{Q} \Phi_{15}+\widetilde{y}_{\mathbf{1 5}}^{Q} \widetilde{\Phi}_{15}\right) Q_{R} \\
& -\overline{L_{L}}\left(y_{1}^{L} \Phi_{1}+\widetilde{y}_{1}^{L} \widetilde{\Phi}_{1}\right) L_{R}+\sqrt{\frac{3}{8}} \overline{L_{L}}\left(y_{15}^{L} \Phi_{15}+\widetilde{y}_{15}^{L} \widetilde{\Phi}_{15}\right) L_{R} \\
& -y_{\Delta} \overline{L_{R}^{c}} \Delta L_{R}+\text { h.c. }
\end{aligned}
$$

where $c$ denotes the charge conjugation and $\widetilde{\Phi}_{\mathbf{1 , 1 5}} \equiv \tau_{2} \Phi_{\mathbf{1 , 1 5}}^{*} \tau_{2}$. Here, we note that $y_{\mathbf{1}, 15}^{Q}=y_{\mathbf{1}, 15}^{L}$ and $\widetilde{y}_{\mathbf{1}, 15}^{Q}=\widetilde{y}_{\mathbf{1}, 15}^{L}$ hold at the first intermediate scale because of the $\mathrm{SU}(4)_{C}$ symmetry. After the bi-doublet fields acquire VEVs, $\left\langle\Phi_{\mathbf{1 , 1 5}}\right\rangle=\operatorname{diag}\left(v_{u}^{\mathbf{1 , 1 5}}, v_{d}^{\mathbf{1 , 1 5}}\right)$, the operators including these VEVs generate a Dirac mass term for the charged SM fields, while the 
VEV of $\Delta$ gives rise to a Majorana mass terms for the right-handed neutrinos. Since both the $\Phi_{1,15}$ and $\Phi_{1,15}^{*}$ can couple to the SM fermions, with different Yukawa couplings, the form of the Yukawa couplings is similar to that of a generic two-Higgs doublet model. ${ }^{7}$ This Yukawa structure in general suffers from large flavor-changing neutral currents [90-92]. ${ }^{8}$ In this work, we just assume that the Yukawa couplings are appropriately aligned so that the flavor-changing processes induced by the exchange of the Higgs fields are sufficiently suppressed.

As mentioned above, the lepton Yukawa couplings are equal to the quark Yukawa couplings at the first intermediate scale. As a result, the Dirac Yukawa couplings for neutrinos are sizable. This is potentially problematic since the Majorana masses for righthanded neutrinos in our model are at the TeV scale. To discuss this point, let us investigate the neutrino mass terms. The masses of light neutrinos in our model are given by the ordinary seesaw relation $[107-112]$,

$$
m_{\nu} \simeq-m_{D} m_{\nu_{R}}^{-1} m_{D}^{T}
$$

with

$$
m_{\nu_{R}}=y_{\Delta} v_{R}^{\mathbf{1 0}}, \quad m_{D}=\left(y_{\mathbf{1}}^{L} v_{u}^{\mathbf{1}}+\widetilde{y}_{\mathbf{1}}^{L} v_{d}^{\mathbf{1}}\right)-\sqrt{\frac{3}{8}}\left(y_{\mathbf{1 5}}^{L} v_{u}^{\mathbf{1 5}}+\widetilde{y}_{15}^{L} v_{d}^{\mathbf{1 5}}\right) .
$$

The first expression in eq. (2.16) induces Majorana masses of $\mathcal{O}\left(v_{R}^{\mathbf{1 0}}\right)$. Then, if the lepton Yukawa couplings are as large as the quark Yukawa couplings, $m_{D}$ becomes as large as quark masses and thus light neutrino masses generically become much larger than $\mathcal{O}(1) \mathrm{eV}$. To get small neutrino masses, in this paper, we suppress $m_{D}$ by fine-tuning the lepton Yukawa couplings. Notice that this fine-tuning is possible because the VEV of the $(\mathbf{1 5}, \mathbf{2}, \mathbf{2})$ component breaks both the $\mathrm{SU}(4)_{C}$ and $\mathrm{SU}(2)_{R}$ relations [113-116]. The breaking of the $\mathrm{SU}(4)_{C}$ relation can be seen in the different factors in front of $y_{15}^{Q}$ and $y_{15}^{L}$, while the breaking of the $\mathrm{SU}(2)_{R}$ relation is realized when $v_{u}^{\mathbf{1 5}} \neq v_{d}^{\mathbf{1 5}}$. This allows us to choose the neutrino Dirac Yukawa couplings freely. In this sense, the $(\mathbf{1 5}, \mathbf{2}, \mathbf{2})$ component is a necessary ingredient to make this model viable. ${ }^{9}$

As we discuss below, we take right-handed neutrino masses to be larger than $m_{W_{R}}$ to forbid the leptonic decay channels of $W_{R}^{ \pm}$. On the other hand, the right-handed neutrino masses are induced by the Yukawa coupling $y_{\Delta}$. To satisfy this condition with a perturbative $y_{\Delta}$, we have a lower bound on $v_{R}^{\mathbf{1 0}}$, which then implies a lower bound on the $Z_{R}$ mass, as can be seen from eq. (2.7). For instance, for $m_{W_{R}}=1.9 \mathrm{TeV}$ and $y_{\Delta}<1$, we have $m_{Z_{R}}>2.2 \mathrm{TeV}$. The gray shaded region in figure 2 shows the area that results in small right-handed neutrino masses. As can be seen from this figure, the LHC constraints on $M_{Z_{R}}$ are more severe.

Before concluding this section, we briefly discuss constraints on $W_{R}$ from flavor observables. The presence of $W_{R}$ in general induces additional flavor violation. Currently,

\footnotetext{
${ }^{7}$ Although there are 4 Higgs doublets below the $\mathrm{TeV}$ scale contributing to electroweak symmetry breaking, the $\mathrm{SU}(2)_{R}$ symmetry reduces the number of Yukawa couplings.

${ }^{8}$ However, such a generic flavor structure may explain a $2.4 \sigma$ excess in the $h \rightarrow \mu \tau$ decay process recently reported by the CMS collaboration [93]. For interpretations of this excess based on the generic two-Higgs doublet model, see refs. [94-106].

${ }^{9}$ The same trick can be used to resolve discrepancies among the charged lepton and quark mass relations.
} 
the $K_{L}-K_{S}$ mass difference gives the most severe constraint on the mass of $W_{R}$, which is roughly given by [117-119]

$$
m_{W_{R}} \gtrsim\left(\frac{g_{2 R}}{g_{2 L}}\right) \times 2.5 \mathrm{TeV} \simeq 1.7 \mathrm{TeV}
$$

where we have set $g_{2 R} / g_{2 L} \simeq 0.67$, which is predicted in our model (see eq. (2.6)). Hence, $\mathrm{a} \sim 2 \mathrm{TeV} W_{R}$ is still allowed by flavor bounds.

\section{Diboson signal}

The ATLAS collaboration has reported an anomalous excess of events in the search for resonances decaying into a pair of gauge bosons, focussing on the hadronically decaying gauge bosons [23]. These gauge bosons are detected as a fat jet since they come from a heavy resonance and are highly boosted and thus quarks from the gauge boson are reconstructed as a single jet with a large jet radius. By investigating invariant mass distributions constructed from two fat jets, the ATLAS collaboration has observed a narrow resonance around $2 \mathrm{TeV}$ with $W Z, W W$, and $Z Z$ final states having a local significance of $3.4,2.6$, and $2.9 \sigma$, respectively. ${ }^{10}$ The tagging selections for each channel are inexact so that sizable events are expected to contaminate other channels. The CMS collaboration also performed a similar search with no discrimination between $W$ and $Z$ bosons, and found a small excess around $1.9 \mathrm{TeV}$ [122]. Recently, the ATLAS collaboration provided an analysis [123] that combines searches for diboson resonances decaying into fully leptonic [124], semi-leptonic [125, 126], and hadronic final states [23]. This still exhibits a $2.5 \sigma$ deviation around $2 \mathrm{TeV}$ in the $W Z$ channel. The CMS experiment has also presented their semi-leptonic search result and found a slight excess around $1.8 \mathrm{TeV}$ [127], although the collaboration found no deviation for the leptonic decay channel [128]. In light of these results, we here discuss the possibility that $W_{R}^{ \pm}$in our model accounts for the excesses observed in the above searches.

The $W_{R}^{ \pm}$can decay into $W^{ \pm}$and $Z$ through mixing with $W^{ \pm}$, and thus can yield diboson signals [24-45]. In addition, it can decay into a pair of quarks as well as $W^{ \pm} h$. If right-handed neutrinos are lighter than the $W_{R}^{ \pm}$mass, the $\ell_{R}^{ \pm} \nu_{R}$ channels are also open, otherwise the $W_{R}^{ \pm}$does not have leptonic decay channels. The dijet, third-generationquark, and $W^{ \pm} h$ resonance searches are also important for determining if $W_{R}^{ \pm}$can explain the diboson anomaly. As for the dijet resonance searches, the CMS experiment [129] observed $\mathrm{a} \sim 2 \sigma$ excess around $1.8 \mathrm{TeV}$, while the ATLAS collaboration found no significant excess around this region [130]. The CMS collaboration also reported a $2.2 \sigma$ excess around $1.8 \mathrm{TeV}$ in the $W^{ \pm} h$ channel, where the $W^{ \pm}$decays leptonically and the Higgs boson $h$ decays to $b \bar{b}$ [131]. However, the ATLAS collaboration did not observe any deviation from the background [132]. The CMS experiment also analyzed the $W^{ \pm} h$ final states in the fully hadronic channel [133] and found no significant excess. Finally, the searches in the top and bottom quark final states exhibit no excess around $\sim 2 \mathrm{TeV}$ [134-137].

\footnotetext{
${ }^{10}$ We note that possible subtleties in this ATLAS analysis have been pointed out in ref. [120]. For a subsequent study on this fat-jet analysis performed by the ATLAS collaboration, see ref. [121].
} 
Considering these results, the authors in ref. [43] carried out a cross-section fit to the data for a resonance in the vicinity of $1.8-1.9 \mathrm{TeV}$; the resultant cross sections they obtained are $^{11}$

$$
\begin{aligned}
\sigma\left(p p \rightarrow W_{R} \rightarrow W Z\right) & =5.7_{-3.3}^{+3.6} \mathrm{fb}, \\
\sigma\left(p p \rightarrow W_{R} \rightarrow W h\right) & =4.5_{-4.0}^{+5.2} \mathrm{fb}, \\
\sigma\left(p p \rightarrow W_{R} \rightarrow j j\right) & =91_{-45}^{+53} \mathrm{fb}, \\
\sigma\left(p p \rightarrow W_{R} \rightarrow t b\right) & =0_{-0}^{+39} \mathrm{fb} .
\end{aligned}
$$

In our model, the production cross section of $W_{R}^{ \pm}$is given as a function of the $W_{R}^{ \pm}$mass, $m_{W_{R}}$, and the $\mathrm{SU}(2)_{R}$ gauge coupling, $g_{2 R}$. The former is determined by the position of the resonance, while the latter is determined by enforcing gauge coupling unification and is given in eqs. (2.6). The partial decay widths of the dijet and $t b$ channels are also determined by these two quantities. On the other hand, the partial decay widths of the $W Z$ and $W h$ channels depend on the $W-W_{R}$ mixing angle $\phi_{L R}^{W}$ defined by,

$$
\left(\begin{array}{l}
W_{L} \\
W_{R}
\end{array}\right)=\left(\begin{array}{cc}
\cos \phi_{L R}^{W} & -\sin \phi_{L R}^{W} \\
\sin \phi_{L R}^{W} & \cos \phi_{L R}^{W}
\end{array}\right)\left(\begin{array}{c}
W \\
W^{\prime}
\end{array}\right)
$$

The size of the mixing angle is expected to be $\phi_{L R}^{W}=\mathcal{O}\left(m_{W}^{2} / m_{W_{R}}^{2}\right) \sim 10^{-3}$, though it is highly dependent on the Higgs sector of the model. In the minimal left-right symmetric model [74-77], which is the same as $v_{R}^{\mathbf{1 5}}=0$ in our model, the mixing angle is given by

$$
\phi_{L R}^{W} \simeq \sin 2 \beta\left(\frac{g_{2 R}}{g_{2 L}}\right) \frac{m_{W}^{2}}{m_{W_{R}}^{2}},
$$

where $\tan \beta$ is the ratio of the VEVs of the bi-doublet Higgs field $(\mathbf{1}, \mathbf{2}, \mathbf{2})_{C}$. To explain the observed top and bottom quark mass ratio, $\tan \beta \simeq m_{t} / m_{b}$ is usually favored in this model. In our model, on the other hand, both $\Phi_{1}$ and $\Phi_{15}$ contribute to the mixing angle with a similar form to the above expression. Contrary to the minimal left-right symmetric case, the VEVs of these fields can be chosen almost arbitrary thanks to the generic structure of the Yukawa sector; we can always reproduce the quark and lepton masses by appropriately choosing these VEVs and Yukawa couplings. Because of this added freedom in our model, we do not specify the Higgs and Yukawa sectors of our models and regard the mixing angle as just a free parameter of order $10^{-3}$. To simplify our discussion, we also assume the righthanded neutrino masses are larger than the $W_{R}$ mass in order to forbid the $\ell_{R}^{ \pm} \nu_{R}$ decay channels. At the end of this section, we briefly discuss the case in which a right-handed neutrino has a mass lighter than $m_{W_{R}}$.

\footnotetext{
${ }^{11}$ Here, we show the result for $\sigma\left(p p \rightarrow W_{R} \rightarrow t b\right)$ without using the ATLAS $\ell \nu b \bar{b}$ result [135]. As discussed in ref. [43], this ATLAS search systematically gives limits on the $W_{R}$ cross sections which are about $2 \sigma$ smaller than the expected limit for the entire mass range consider. This leads to a strong upper limit on $\sigma\left(p p \rightarrow W_{R} \rightarrow t b\right)$ after the fit of $38 \mathrm{fb}$ which is due to the fitting method used in that work. Considering the possibility of a systematic error in the ATLAS $\ell \nu b \bar{b}$ search with its large effect on the cross section fit shown in ref. [43], we use the cross sections found when the ATLAS $\ell \nu b \bar{b}$ search is ignored.
} 


\begin{tabular}{|c|c|cccc|}
\hline $\sin \phi_{L R}^{W}$ & $\Gamma_{\text {tot }}[\mathrm{GeV}]$ & $\mathrm{BR}(j j)$ & $\mathrm{BR}(t b)$ & $\mathrm{BR}(W Z)$ & $\mathrm{BR}(W h)$ \\
\hline 0.0016 & 22.3 & 0.61 & 0.30 & 0.047 & 0.046 \\
0.0012 & 21.4 & 0.63 & 0.31 & 0.027 & 0.027 \\
\hline
\end{tabular}

Table 2. Total decay width $\Gamma_{\text {tot }}$ and the branching ratios of $W_{R}^{ \pm}$for our model. Here, we set $m_{W_{R}}=1.9 \mathrm{TeV}$ and use the gauge couplings given in eq. (2.6).

The authors in ref. [43] performed a parameter fitting in an $\mathrm{SU}(2)_{L} \otimes \mathrm{SU}(2)_{R} \otimes \mathrm{U}(1)_{B-L}$ model to determine the model parameters, $g_{2 R} / g_{2 L}$ and $\phi_{L R}^{W}$, for which the required cross sections given above are satisfied. Since the $W_{R}$ sector in our model is the same as that in ref. [43], we can directly apply their result to our case. According to their $68 \%$ CL result,

$$
0.5 \lesssim g_{2 R} / g_{2 L} \lesssim 0.65, \quad 0.0012 \lesssim \sin \phi_{L R}^{W} \lesssim 0.0016
$$

where they took $m_{W_{R}}=1.9 \mathrm{TeV}$, the value preferred by the current experimental data. Notice that our model predicts $g_{2 R} / g_{2 L} \simeq 0.67$ as can be seen in eqs. (2.6), and is very close to the preferred region. Therefore, by choosing the mixing angle in the range presented in eq. (3.7), our model can explain the ATLAS diboson anomaly without conflicting with other collider bounds.

Using the gauge couplings given in eq. (2.6), and a mixing angle in the range found in eq. (3.7), we evaluate the production cross section $\sigma\left(p p \rightarrow W_{R}^{ \pm}\right)$, total decay width $\Gamma_{\text {tot }}$, and the branching ratios of $W_{R}^{ \pm}$which are given in the appendix. The total decay width and branching fractions for our model are summarized in table 2. Here, we set $m_{W_{R}}=1.9 \mathrm{TeV}$. Notice that $\operatorname{BR}(W Z)$ is sizable even though the mixing angle $\sin \phi_{L R}^{W}$ is very small. This is because the $W_{R}^{ \pm} \rightarrow W^{ \pm} Z$ decay process is enhanced by a factor of $\left(m_{W_{R}} / m_{W}\right)^{4}$ due to the high-energy behavior of the longitudinal mode of $W_{R}^{ \pm}$. This enhancement compensates for the suppression coming from the mixing angle. In addition, we have $\mathrm{BR}(W Z) \simeq \mathrm{BR}(W h)$, as a consequence of the equivalence theorem. As for the production cross section, we compute it using MADGRAPH5 [138] at the leading order, and re-scale by the so-called $k$ factor which corresponds to the effects of the higher-order corrections; this value is found to be $k \simeq 1.3[139,140]$. The resultant cross section is

$$
\sigma\left(p p \rightarrow W_{R}^{ \pm}\right) \simeq 143 \times\left(\frac{g_{2 R}}{0.42}\right)^{2} \mathrm{fb},
$$

again for $m_{W_{R}}=1.9 \mathrm{TeV}$. Considering the fact that about a half of $W Z$ events decay hadronically, and using the acceptance $\sim 0.14$ [23], we estimate that there are about $9(5)$ additional events in total in the presence of $W_{R}^{ \pm}$if $\sin \phi_{L R}^{W}=0.0016(0.0012)$. This can explain the diboson excess observed in ref. [23].

As we have seen above, to explain the diboson anomaly, the mixing angle $\phi_{L R}^{W}$ should be $\mathcal{O}\left(10^{-3}\right)$. This size of the $W-W_{R}$ mixing could be constrained by the electroweak precision measurements [24, 42]. Indeed, the authors in ref. [141] derived an upper limit on the mixing angle from the bound on the $T$-parameter of $\left|\phi_{L R}^{W}\right| \lesssim 5 \times 10^{-4}$ for $m_{W_{R}}=2 \mathrm{TeV}$, which is smaller than the values in eq. (3.7). However, our model also contains $Z_{R}$, which affects the electroweak observables as well. Moreover, since its contribution modifies the 
$Z$-boson coupling to the SM fermions at tree level through the $Z-Z_{R}$ mixing, we cannot use the conventional method relying on the $S$ and $T$ parameters $[142,143]$ to evaluate the electroweak precision constraints. Instead, we need to perform a complete parameter fitting onto the electroweak observables. This is beyond the scope of this paper. We however note that such a parameter fitting has been carried out for the $\mathrm{SU}(2)_{L} \otimes \mathrm{SU}(2)_{R} \otimes \mathrm{U}(1)_{B-L}$ models in the literature $[139,144]$ and, according to the results, a $2 \mathrm{TeV} W_{R}$ with an $\mathcal{O}\left(10^{-3}\right) W-W_{R}$ mixing can evade the electroweak precision constraint. Since the structure of the $\mathrm{TeV}$-scale gauge sector of our model is similar to those in these models, we expect that our model can also avoid this constraint.

Next, we consider the case where a right-handed neutrino has a mass lighter than the $W_{R}$ mass. This case is potentially interesting since it may explain the $2.8 \sigma$ anomaly observed by the CMS collaboration in the right-handed neutrino searches looking for dijet plus dilepton events [46]. Indeed, it turns out that $W_{R}$ with $g_{2 R} / g_{2 L} \simeq 0.6$ and $m_{W_{R}} \sim$ $2 \mathrm{TeV}$ may explain the excess if the $W_{R}$ decays into a right-handed neutrino that mainly couples to first generation charged leptons [145-147], i.e. $W_{R}^{ \pm} \rightarrow \ell^{ \pm} N_{R} \rightarrow \ell^{ \pm} \ell^{ \pm} j j$. This simple setup is, however, disfavored once the charge of observed electrons/positrons is considered. The CMS collaboration observed only one same-sign electron event on top of the 13 opposite-sign events. However, if the right-handed neutrino is a Majorana fermion, we expect almost the same numbers of events for each case. ${ }^{12}$ In addition, such a Majorana right-handed neutrino is severely restricted by the ATLAS search for same-sign leptons plus dijet events [148]. To reconcile these experimental results, there are several possibilities for extending the neutrino sector that work, such as the inverse seesaw $[149,150]$ or the linear seesaw [151-153] mechanisms which were discussed in the context of the diboson anomaly in refs. [44] and [45], respectively. In these cases, heavy neutrinos become pseudo-Dirac fermions, which allows them to evade the bounds from the same-sign leptons plus dijet searches $[46,148]$. Although our model potentially accommodates such an extension, we leave the investigation of this to future work.

Finally, we discuss future prospects for testing this model. The first step for verifying our model is, of course, to confirm the presence of a $\sim 2 \mathrm{TeV} W_{R}$ at Run-II of the LHC. The production cross section of a $W_{R}$ in our model at a $13 \mathrm{TeV}$ center-of-mass energy is estimated as $\sim 800 \mathrm{fb}$, where we have used the $k$ factor of $k \simeq 1.2[139,140]$. Therefore, a $\sim 2 \mathrm{TeV} W_{R}$ can be tested with an integrated luminosity of $\sim 10 \mathrm{fb}^{-1}$. As discussed above, our model may predict a relatively light $Z_{R}$, which may also be reached at the LHC. In addition, a (fat) jet plus missing energy search can also probe this model [154], which would constrain the decay mode $W Z \rightarrow \nu \bar{\nu} j j$.

The presence of an $\mathrm{SU}(2)_{L}$ triplet Dirac fermion dark matter is a distinct feature of our model, and thus dark matter searches can also play an important role in testing this model. Since we expect its mass to be around $2 \mathrm{TeV}$, it is difficult for the LHC to probe it directly. Instead, indirect dark matter detection experiments, such as searches for gamma rays from the Galactic Center or dwarf spheroidal galaxies, are quite promising

\footnotetext{
${ }^{12}$ Since a Majorana fermion and its anti-particle are identical, if a Majorana fermion decays into an electron it would also decay into a positron with the same rate.
} 
since this dark matter has a relatively large annihilation cross section. Most work to date on an $\mathrm{SU}(2)_{L}$ triplet fermion dark matter candidate has assumed a Majorana rather than a Dirac particle [155-158] and there is little work on the Dirac triplet dark matter. To test the dark matter in our model in the indirect detection experiments, it is quite important to evaluate its annihilation cross section precisely and compare it with that of the Majorana dark matter. The direct detection rate of the $\mathrm{SU}(2)_{L}$ triplet dark matter is rather small; its scattering cross section with a nucleon is computed as $\sigma_{\mathrm{SI}} \simeq 2 \times 10^{-47} \mathrm{~cm}^{2}$ [159]. Still, it is in principle detectable in future experiments, which gives another way to test our model.

\section{Conclusion}

We have considered a specific (non-supersymmetric) $\mathrm{SO}(10)$ model which can simultaneously explain the purported ATLAS diboson excess, provide a stable dark matter candidate, and achieve gauge coupling unification with a suitably high GUT scale. In addition to the gauge and matter sector of the theory which are standard for $\mathrm{SO}(10)$, the model has Higgs fields with three different representations under the $\mathrm{SO}(10)$ gauge symmetry: a $\mathbf{2 1 0}$ which is used to break $\mathrm{SO}(10)$, and plays an role in breaking both of the intermediate scale gauge groups, $\mathrm{SU}(4)_{C} \otimes \mathrm{SU}(2)_{L} \otimes \mathrm{SU}(2)_{R}$ and $\mathrm{SU}(3)_{C} \otimes \mathrm{SU}(2)_{L} \otimes \mathrm{SU}(2)_{R} \otimes \mathrm{U}(1)_{B-L} ;$ a 126 which assist the breaking of the smaller intermediate gauge group and participates in the breaking of the SM gauge symmetries; a 10 which is also involved in the breaking of the SM gauge symmetries. The model also introduces a Dirac $\mathbf{4 5}$ which provides us with a stable dark matter candidate in the form of a $\mathrm{SU}(2)_{L}$ triplet. Having fixed the lower intermediate scale at $1.9 \mathrm{TeV}$ (to account for the diboson excess), the GUT scale of order $10^{17} \mathrm{GeV}$ and the high intermediate scale of order $10^{9} \mathrm{GeV}$ are determined by requiring gauge coupling unification. In this model, both stages of intermediate symmetry breaking as well as a $\mathrm{TeV}$-scale dark matter triplet are necessary for unification of the gauge couplings.

The high GUT scale ensures proton stability. The relatively high intermediate scale of $10^{9} \mathrm{GeV}$, ensures that the effects induced by the presence of both vector and scalar leptoquarks are small enough to remain compatible with experimental constraints. The lower intermediate scale was chosen to account for the diboson excess, and predicts new right-handed gauge bosons to appear at the $\mathrm{TeV}$ scale. In addition to a charged $W_{R}^{ \pm}$pair, the model predicts a new $Z_{R}$ gauge boson whose mass depends on the relative contributions of the two Higgs VEVs participating in the breaking of the low intermediate scale. This set of $\mathrm{TeV}$ scale gauge bosons should allow the model to be tested in current and future runs at the LHC.

The contributions of two Higgs VEVs to SM breaking gives the model the flexibility needed to adjust the SM fermion spectrum, thus avoiding some of the overly restrictive predictions often encountered in minimal $\mathrm{SO}(10)$ models. This is necessary if one hopes to derive $\mathrm{eV}$ scale neutrino masses from the seesaw mechanism given that the Majorana mass for the right-handed neutrino is also at the TeV scale.

Finally, the model contains a testable dark matter candidate and the neutral member of an electroweak triplet. Similar to the often studied supersymmetric wino, the mass splitting is rather small (typically of order $165 \mathrm{MeV}$ ), and the annihilation cross section is 
rather large, requiring the mass of the triplet to be of order $2 \mathrm{TeV}$ as well. Thus the dark matter candidate in this model is testable in indirect dark matter searches as well as direct detection experiments.

Note added. While completing this work, the CMS collaboration announced a new result for the dijet resonance search using the $13 \mathrm{TeV}$ LHC data with an integrated luminosity of $2.4 \mathrm{fb}^{-1}[160]$. They give an upper bound on the product of the production cross section $(\sigma)$, the branching fraction of the dijet channel $(\operatorname{Br}(j j))$, and acceptance $(A)$ of $\sigma A \operatorname{Br}(j j) \lesssim$ $400 \mathrm{fb}$ for a resonance mass of $1.9 \mathrm{TeV}$, and thus we have $\sigma \operatorname{Br}(j j) \lesssim 667 \mathrm{fb}$ using $A \simeq$ 0.6 [160]. The ATLAS collaboration also reported a $13-\mathrm{TeV}$ result for the same channel based on the $3.6 \mathrm{fb}^{-1}$ data, and gave a more severe constraint [161]: $\sigma A \operatorname{Br}(j j) \lesssim 210 \mathrm{fb}$ for a $1.9 \mathrm{TeV}$ resonance. By using $A \simeq 0.4$ [161], we obtain $\sigma \operatorname{Br}(j j) \lesssim 525 \mathrm{fb}$. On the other hand, our model predicts $\sigma \operatorname{Br}(j j)$ to be $\sigma \operatorname{Br}(j j)=421$ (485) fb for a $1.9 \mathrm{TeV} W_{R}$ with $\sin \phi_{L R}^{W}=0.0016(0.0012)$, and thus evades these limits. Since these limits are fairly close to the model predictions, we expect that the presence of $W_{R}$ in our model can be confirmed in near future.

\section{Acknowledgments}

The work of J.L.E., N.N. and K.A.O. was supported by DOE grant DE-SC0011842 at the University of Minnesota.

\section{A Decay widths}

Here, we summarize the partial decay widths of $W_{R}$ we use in our calculation. For the fermionic channels, $W_{R} \rightarrow f \bar{f}^{\prime}$, we use

$$
\begin{aligned}
\Gamma\left(W_{R}^{+} \rightarrow u \bar{d}\right) & =\Gamma\left(W_{R}^{+} \rightarrow c \bar{s}\right)=\frac{g_{2 R}^{2}}{16 \pi} m_{W_{R}} \\
\Gamma\left(W_{R}^{+} \rightarrow t \bar{b}\right) & =\frac{g_{2 R}^{2}}{16 \pi} m_{W_{R}}\left(1+\frac{m_{t}^{2}}{2 m_{W_{R}}^{2}}\right)\left(1-\frac{m_{t}^{2}}{m_{W_{R}}^{2}}\right)^{2} .
\end{aligned}
$$

For the $W_{R} \rightarrow W Z$ decay process, we have

$$
\begin{aligned}
\Gamma\left(W_{R}^{+} \rightarrow W^{+} Z\right)= & \frac{g_{2 L}^{2}}{192 \pi} \sin ^{2} \phi_{L R}^{W} \frac{m_{W_{R}}^{5}}{m_{W}^{4}}\left(1-2 \frac{m_{W}^{2}+m_{Z}^{2}}{m_{W_{R}}^{2}}+\frac{\left(m_{W}^{2}-m_{Z}^{2}\right)^{2}}{m_{W_{R}}^{4}}\right)^{\frac{3}{2}} \\
& \times\left(1+10 \frac{m_{W}^{2}+m_{Z}^{2}}{m_{W_{R}}^{2}}+\frac{m_{W}^{4}+10 m_{W}^{2} m_{Z}^{2}+m_{Z}^{4}}{m_{W_{R}}^{4}}\right) .
\end{aligned}
$$

Finally, the $W_{R} \rightarrow W h$ decay width is given by

$$
\begin{aligned}
\Gamma\left(W_{R}^{+} \rightarrow W^{+} h\right)= & \frac{g_{2 L}^{2}}{192 \pi} \sin ^{2} \phi_{L R}^{W} \frac{m_{W_{R}}^{5}}{m_{W}^{4}}\left(1-2 \frac{m_{W}^{2}+m_{h}^{2}}{m_{W_{R}}^{2}}+\frac{\left(m_{W}^{2}-m_{h}^{2}\right)^{2}}{m_{W_{R}}^{4}}\right)^{\frac{1}{2}} \\
& \times\left(1+\frac{10 m_{W}^{2}-2 m_{h}^{2}}{m_{W_{R}}^{2}}+\frac{\left(m_{W}^{2}-m_{h}^{2}\right)^{2}}{m_{W_{R}}^{4}}\right)
\end{aligned}
$$

in the decoupling limit. 
Open Access. This article is distributed under the terms of the Creative Commons Attribution License (CC-BY 4.0), which permits any use, distribution and reproduction in any medium, provided the original author(s) and source are credited.

\section{References}

[1] H. Georgi and S.L. Glashow, Unity of All Elementary Particle Forces, Phys. Rev. Lett. 32 (1974) 438 [INSPIRE].

[2] H. Georgi, The State of the Art-Gauge Theories, AIP Conf.Proc. 23 (1975) 575 [InSPIRE].

[3] H. Fritzsch and P. Minkowski, Unified Interactions of Leptons and Hadrons, Annals Phys. 93 (1975) 193 [inSPIRE].

[4] M.S. Chanowitz, J.R. Ellis and M.K. Gaillard, The Price of Natural Flavor Conservation in Neutral Weak Interactions, Nucl. Phys. B 128 (1977) 506 [inSPIRE].

[5] H. Georgi and D.V. Nanopoulos, Ordinary Predictions from Grand Principles: T Quark Mass in $O(10)$, Nucl. Phys. B 155 (1979) 52 [InSPIRE].

[6] H. Georgi and D.V. Nanopoulos, Masses and Mixing in Unified Theories, Nucl. Phys. B 159 (1979) 16 [INSPIRE].

[7] C.E. Vayonakis, On Mass Relations and Renormalization Effects in Grand Unified Theories, Phys. Lett. B 82 (1979) 224 [Erratum ibid. B83 (1979) 421] [INSPIRE].

[8] S. Rajpoot, Symmetry breaking and intermediate mass scales in the $\mathrm{SO}(10)$ grand unified theory, Phys. Rev. D 22 (1980) 2244 [InSPIRE].

[9] M. Yasue, Phenomenological Aspect of SO(10) Grand Unified Model, Prog. Theor. Phys. 65 (1981) 708 [Erratum ibid. 65 (1981) 1480] [INSPIRE].

[10] J.M. Gipson and R.E. Marshak, Intermediate Mass Scales in the New $\mathrm{SO}(10)$ Grand Unification in the One Loop Approximation, Phys. Rev. D 31 (1985) 1705 [InSPIRE].

[11] D. Chang, R.N. Mohapatra, J. Gipson, R.E. Marshak and M.K. Parida, Experimental Tests of New SO(10) Grand Unification, Phys. Rev. D 31 (1985) 1718 [inSPIRE].

[12] N.G. Deshpande, E. Keith and P.B. Pal, Implications of LEP results for $\mathrm{SO}(10)$ grand unification, Phys. Rev. D 46 (1993) 2261 [INSPIRE].

[13] N.G. Deshpande, E. Keith and P.B. Pal, Implications of LEP results for $\mathrm{SO}(10)$ grand unification with two intermediate stages, Phys. Rev. D 47 (1993) 2892 [hep-ph/9211232] [INSPIRE].

[14] S. Bertolini, L. Di Luzio and M. Malinsky, On the vacuum of the minimal nonsupersymmetric SO(10) unification, Phys. Rev. D 81 (2010) 035015 [arXiv:0912.1796] [INSPIRE].

[15] M. Fukugita and T. Yanagida, Physics of neutrinos, in Physics and astrophysics of neutrinos, M. Fukugita and A. Suzuki eds., pg. 1-248, Kyoto University, YITP-K-1050 (93/12,rec.Feb.94) pg. 248.

[16] L. Di Luzio, Aspects of symmetry breaking in Grand Unified Theories, arXiv:1110.3210 [INSPIRE].

[17] T. Fukuyama, SO(10) GUT in Four and Five Dimensions: A Review, Int. J. Mod. Phys. A 28 (2013) 1330008 [arXiv: 1212.3407] [INSPIRE]. 
[18] A. Masiero, On the Phenomenological Group in Unified SO(10) Model, Phys. Lett. B 93 (1980) 295 [INSPIRE].

[19] G. Lazarides, Q. Shafi and C. Wetterich, Proton Lifetime and Fermion Masses in an SO(10) Model, Nucl. Phys. B 181 (1981) 287 [inSPIRE].

[20] Q. Shafi, M. Sondermann and C. Wetterich, Fourth Color in O(10), Phys. Lett. B 92 (1980) 304 [INSPIRE].

[21] F. del Aguila and L.E. Ibáñez, Higgs Bosons in SO(10) and Partial Unification, Nucl. Phys. B 177 (1981) 60 [INSPIRE].

[22] K.S. Babu and S. Khan, Minimal nonsupersymmetric SO(10) model: Gauge coupling unification, proton decay and fermion masses, Phys. Rev. D 92 (2015) 075018 [arXiv: 1507.06712] [INSPIRE].

[23] ATLAS collaboration, Search for high-mass diboson resonances with boson-tagged jets in proton-proton collisions at $\sqrt{s}=8 \mathrm{TeV}$ with the ATLAS detector, JHEP 12 (2015) 055 [arXiv: 1506.00962] [INSPIRE].

[24] J. Hisano, N. Nagata and Y. Omura, Interpretations of the ATLAS Diboson Resonances, Phys. Rev. D 92 (2015) 055001 [arXiv:1506.03931] [INSPIRE].

[25] K. Cheung, W.-Y. Keung, P.-Y. Tseng and T.-C. Yuan, Interpretations of the ATLAS Diboson Anomaly, Phys. Lett. B 751 (2015) 188 [arXiv:1506.06064] [InSPIRE].

[26] B.A. Dobrescu and Z. Liu, $W^{\prime}$ Boson near 2 TeV: Predictions for Run 2 of the LHC, Phys. Rev. Lett. 115 (2015) 211802 [arXiv:1506.06736] [INSPIRE].

[27] A. Thamm, R. Torre and A. Wulzer, Composite Heavy Vector Triplet in the ATLAS Diboson Excess, Phys. Rev. Lett. 115 (2015) 221802 [arXiv:1506.08688] [InSPIRE].

[28] Q.-H. Cao, B. Yan and D.-M. Zhang, Simple non-Abelian extensions of the standard model gauge group and the diboson excesses at the LHC, Phys. Rev. D 92 (2015) 095025 [arXiv: 1507.00268] [INSPIRE].

[29] T. Abe, R. Nagai, S. Okawa and M. Tanabashi, Unitarity sum rules, three-site moose model and the ATLAS 2 TeV diboson anomalies, Phys. Rev. D 92 (2015) 055016 [arXiv: 1507.01185] [INSPIRE].

[30] B.C. Allanach, B. Gripaios and D. Sutherland, Anatomy of the ATLAS diboson anomaly, Phys. Rev. D 92 (2015) 055003 [arXiv: 1507.01638] [INSPIRE].

[31] T. Abe, T. Kitahara and M.M. Nojiri, Prospects for Spin-1 Resonance Search at $13 \mathrm{TeV}$ LHC and the ATLAS Diboson Excess, arXiv:1507.01681 [INSPIRE].

[32] B.A. Dobrescu and Z. Liu, Heavy Higgs bosons and the $2 \mathrm{TeV} W^{\prime}$ boson, JHEP 10 (2015) 118 [arXiv: 1507.01923] [INSPIRE].

[33] A.E. Faraggi and M. Guzzi, Extra $Z^{\prime} s$ and $W^{\prime} s$ in heterotic-string derived models, Eur. Phys. J. C 75 (2015) 537 [arXiv:1507.07406] [INSPIRE].

[34] P. Coloma, B.A. Dobrescu and J. Lopez-Pavon, Right-handed neutrinos and the $2 \mathrm{TeV} W^{\prime}$ boson, Phys. Rev. D 92 (2015) 115023 [arXiv:1508.04129] [INSPIRE].

[35] J.H. Collins and W.H. Ng, A $2 \mathrm{TeV} W_{R}$, supersymmetry and the Higgs mass, JHEP 01 (2016) 159 [arXiv: 1510.08083] [INSPIRE].

[36] B.A. Dobrescu and P.J. Fox, Signals of a $2 T e V W^{\prime}$ boson and a heavier $Z^{\prime}$ boson, arXiv:1511.02148 [INSPIRE]. 
[37] A. Sajjad, Understanding diboson anomalies, arXiv:1511.02244 [INSPIRE].

[38] T. Appelquist, Y. Bai, J. Ingoldby and M. Piai, Spectrum-doubled Heavy Vector Bosons at the LHC, JHEP 01 (2016) 109 [arXiv:1511.05473] [INSPIRE].

[39] K. Das, T. Li, S. Nandi and S.K. Rai, Diboson excesses in an anomaly free leptophobic left-right model, Phys. Rev. D 93 (2016) 016006 [arXiv:1512.00190] [INSPIRE].

[40] J.A. Aguilar-Saavedra and F.R. Joaquim, Multiboson production in $W^{\prime}$ decays, JHEP 01 (2016) 183 [arXiv:1512.00396] [InSPIRE].

[41] M. Hirsch, M.E. Krauss, T. Opferkuch, W. Porod and F. Staub, A constrained supersymmetric left-right model, arXiv:1512.00472 [INSPIRE].

[42] Y. Gao, T. Ghosh, K. Sinha and J.-H. Yu, SU(2) $\times \mathrm{SU}(2) \times \mathrm{U}(1)$ interpretations of the diboson and Wh excesses, Phys. Rev. D 92 (2015) 055030 [arXiv:1506.07511] [INSPIRE].

[43] J. Brehmer, J. Hewett, J. Kopp, T. Rizzo and J. Tattersall, Symmetry Restored in Dibosons at the LHC?, JHEP 10 (2015) 182 [arXiv:1507.00013] [INSPIRE].

[44] P.S. Bhupal Dev and R.N. Mohapatra, Unified explanation of the eejj, diboson and dijet resonances at the LHC, Phys. Rev. Lett. 115 (2015) 181803 [arXiv:1508.02277] [INSPIRE].

[45] F.F. Deppisch et al., Reconciling the $2 \mathrm{TeV}$ excesses at the LHC in a linear seesaw left-right model, Phys. Rev. D 93 (2016) 013011 [arXiv: 1508.05940] [InSPIRE].

[46] CMS collaboration, Search for heavy neutrinos and $\mathrm{W}$ bosons with right-handed couplings in proton-proton collisions at $\sqrt{s}=8 \mathrm{TeV}$, Eur. Phys. J. C 74 (2014) 3149 [arXiv:1407.3683] [INSPIRE].

[47] T.W.B. Kibble, G. Lazarides and Q. Shafi, Strings in SO(10), Phys. Lett. B 113 (1982) 237 [INSPIRE].

[48] L.M. Krauss and F. Wilczek, Discrete Gauge Symmetry in Continuum Theories, Phys. Rev. Lett. 62 (1989) 1221 [INSPIRE].

[49] L.E. Ibáñez and G.G. Ross, Discrete gauge symmetry anomalies, Phys. Lett. B 260 (1991) 291 [INSPIRE].

[50] L.E. Ibáñez and G.G. Ross, Discrete gauge symmetries and the origin of baryon and lepton number conservation in supersymmetric versions of the standard model, Nucl. Phys. B 368 (1992) 3 [INSPIRE].

[51] S.P. Martin, Some simple criteria for gauged R-parity, Phys. Rev. D 46 (1992) 2769 [hep-ph/9207218] [INSPIRE].

[52] M. Kadastik, K. Kannike and M. Raidal, Matter parity as the origin of scalar Dark Matter, Phys. Rev. D 81 (2010) 015002 [arXiv:0903.2475] [INSPIRE].

[53] M. Kadastik, K. Kannike and M. Raidal, Dark Matter as the signal of Grand Unification, Phys. Rev. D 80 (2009) 085020 [Erratum ibid. D 81 (2010) 029903] [arXiv:0907.1894] [INSPIRE].

[54] M. Frigerio and T. Hambye, Dark matter stability and unification without supersymmetry, Phys. Rev. D 81 (2010) 075002 [arXiv:0912.1545] [InSPIRE].

[55] T. Hambye, On the stability of particle dark matter, PoS (IDM2010) 098 [arXiv:1012.4587] [INSPIRE]. 
[56] Y. Mambrini, N. Nagata, K.A. Olive, J. Quevillon and J. Zheng, Dark matter and gauge coupling unification in nonsupersymmetric $\mathrm{SO}(10)$ grand unified models, Phys. Rev. D 91 (2015) 095010 [arXiv: 1502.06929] [INSPIRE].

[57] N. Nagata, K.A. Olive and J. Zheng, Weakly-Interacting Massive Particles in Non-supersymmetric SO(10) Grand Unified Models, JHEP 10 (2015) 193 [arXiv: 1509.00809] [INSPIRE].

[58] C. Arbelaez, R. Longas, D. Restrepo and O. Zapata, Fermion dark matter from $\mathrm{SO}(10)$ GUTs, Phys. Rev. D 93 (2016) 013012 [arXiv: 1509.06313] [InSPIRE].

[59] S.M. Boucenna, M.B. Krauss and E. Nardi, Dark Matter from the vector of $\mathrm{SO}(10)$, arXiv: 1511.02524 [INSPIRE].

[60] G.R. Farrar and P. Fayet, Phenomenology of the Production, Decay and Detection of New Hadronic States Associated with Supersymmetry, Phys. Lett. B 76 (1978) 575 [INSPIRE].

[61] S. Dimopoulos and H. Georgi, Softly Broken Supersymmetry and SU(5), Nucl. Phys. B 193 (1981) 150 [INSPIRE].

[62] S. Weinberg, Supersymmetry at Ordinary Energies. 1. Masses and Conservation Laws, Phys. Rev. D 26 (1982) 287 [inSPIRE].

[63] N. Sakai and T. Yanagida, Proton Decay in a Class of Supersymmetric Grand Unified Models, Nucl. Phys. B 197 (1982) 533 [InSPIRE].

[64] S. Dimopoulos, S. Raby and F. Wilczek, Proton Decay in Supersymmetric Models, Phys. Lett. B 112 (1982) 133 [INSPIRE].

[65] U. Aydemir, D. Minic, C. Sun and T. Takeuchi, Pati-Salam unification from noncommutative geometry and the TeV-scale $W_{R}$ boson, Int. J. Mod. Phys. A 31 (2016) 1550223 [arXiv: 1509.01606] [INSPIRE].

[66] T. Bandyopadhyay, B. Brahmachari and A. Raychaudhuri, Implications of the CMS search for $W_{R}$ on grand unification, JHEP 02 (2016) 023 [arXiv: 1509.03232] [INSPIRE].

[67] U. Aydemir, $\mathrm{SO}(10)$ grand unification in light of recent LHC searches and colored scalars at the TeV-scale, arXiv:1512.00568 [INSPIRE].

[68] S. Davidson, D.C. Bailey and B.A. Campbell, Model independent constraints on leptoquarks from rare processes, Z. Phys. C 61 (1994) 613 [hep-ph/9309310] [InSPIRE].

[69] V.A. Kuzmin and M.E. Shaposhnikov, Baryon Asymmetry of the Universe Versus Left-right Symmetry, Phys. Lett. B 92 (1980) 115 [InSPIRE].

[70] T.W.B. Kibble, G. Lazarides and Q. Shafi, Walls Bounded by Strings, Phys. Rev. D 26 (1982) 435 [INSPIRE].

[71] D. Chang, R.N. Mohapatra and M.K. Parida, Decoupling Parity and SU(2)-R Breaking Scales: A New Approach to Left-Right Symmetric Models, Phys. Rev. Lett. 52 (1984) 1072 [INSPIRE].

[72] D. Chang, R.N. Mohapatra and M.K. Parida, A New Approach to Left-Right Symmetry Breaking in Unified Gauge Theories, Phys. Rev. D 30 (1984) 1052 [INSPIRE].

[73] D. Chang, R.N. Mohapatra, J. Gipson, R.E. Marshak and M.K. Parida, Experimental Tests of New SO(10) Grand Unification, Phys. Rev. D 31 (1985) 1718 [InSPIRE].

[74] J.C. Pati and A. Salam, Lepton Number as the Fourth Color, Phys. Rev. D 10 (1974) 275 [Erratum ibid. D 11 (1975) 703] [INSPIRE]. 
[75] R.N. Mohapatra and J.C. Pati, Left-Right Gauge Symmetry and an Isoconjugate Model of CP-violation, Phys. Rev. D 11 (1975) 566 [INSPIRE].

[76] R.N. Mohapatra and J.C. Pati, A Natural Left-Right Symmetry, Phys. Rev. D 11 (1975) 2558 [INSPIRE].

[77] G. Senjanović and R.N. Mohapatra, Exact Left-Right Symmetry and Spontaneous Violation of Parity, Phys. Rev. D 12 (1975) 1502 [inSPIRE].

[78] Planck collaboration, P.A.R. Ade et al., Planck 2015 results. XIII. Cosmological parameters, arXiv:1502.01589 [INSPIRE].

[79] M. Cirelli, N. Fornengo and A. Strumia, Minimal dark matter, Nucl. Phys. B 753 (2006) 178 [hep-ph/0512090] [INSPIRE].

[80] M. Cirelli, A. Strumia and M. Tamburini, Cosmology and Astrophysics of Minimal Dark Matter, Nucl. Phys. B 787 (2007) 152 [arXiv:0706.4071] [INSPIRE].

[81] M. Cirelli and A. Strumia, Minimal Dark Matter: Model and results, New J. Phys. 11 (2009) 105005 [arXiv:0903.3381] [INSPIRE].

[82] J. Hisano, S. Matsumoto and M.M. Nojiri, Explosive dark matter annihilation, Phys. Rev. Lett. 92 (2004) 031303 [hep-ph/0307216] [INSPIRE].

[83] J. Hisano, S. Matsumoto, M.M. Nojiri and O. Saito, Non-perturbative effect on dark matter annihilation and gamma ray signature from galactic center, Phys. Rev. D 71 (2005) 063528 [hep-ph/0412403] [INSPIRE].

[84] J. Hisano, S. Matsumoto, M. Nagai, O. Saito and M. Senami, Non-perturbative effect on thermal relic abundance of dark matter, Phys. Lett. B 646 (2007) 34 [hep-ph/0610249] [INSPIRE].

[85] ATLAS collaboration, Search for high-mass dilepton resonances in pp collisions at $\sqrt{s}=8$ TeV with the ATLAS detector, Phys. Rev. D 90 (2014) 052005 [arXiv:1405.4123] [INSPIRE].

[86] CMS collaboration, Search for physics beyond the standard model in dilepton mass spectra in proton-proton collisions at $\sqrt{s}=8 \mathrm{TeV}$, JHEP 04 (2015) 025 [arXiv:1412.6302] [INSPIRE].

[87] E. Accomando, A. Belyaev, L. Fedeli, S.F. King and C. Shepherd-Themistocleous, $Z^{\prime}$ physics with early LHC data, Phys. Rev. D 83 (2011) 075012 [arXiv: 1010.6058] [INSPIRE].

[88] S. Godfrey and T. Martin, Z' Discovery Reach at Future Hadron Colliders: A Snowmass White Paper, arXiv:1309.1688 [INSPIRE].

[89] CMS collaboration, emphEvent Display of a Candidate Electron-Positron Pair with an Invariant Mass of 2.9 TeV, CMS-DP-2015-039 (2015).

[90] J.D. Bjorken and S. Weinberg, A Mechanism for Nonconservation of Muon Number, Phys. Rev. Lett. 38 (1977) 622 [inSPIRE].

[91] B. McWilliams and L.-F. Li, Virtual Effects of Higgs Particles, Nucl. Phys. B 179 (1981) 62 [INSPIRE].

[92] O.U. Shanker, Flavor Violation, Scalar Particles and Leptoquarks, Nucl. Phys. B 206 (1982) 253 [INSPIRE].

[93] CMS collaboration, Search for Lepton-Flavour-Violating Decays of the Higgs Boson, Phys. Lett. B 749 (2015) 337 [arXiv: 1502.07400] [INSPIRE]. 
[94] D. Aristizabal Sierra and A. Vicente, Explaining the CMS Higgs flavor violating decay excess, Phys. Rev. D 90 (2014) 115004 [arXiv:1409.7690] [inSPIRE].

[95] A. Crivellin, G. D'Ambrosio and J. Heeck, Explaining $h \rightarrow \mu^{ \pm} \tau^{\mp}, B \rightarrow K^{*} \mu^{+} \mu^{-}$and $B \rightarrow K \mu^{+} \mu^{-} / B \rightarrow K e^{+} e^{-}$in a two-Higgs-doublet model with gauged $L_{\mu}-L_{\tau}$, Phys. Rev. Lett. 114 (2015) 151801 [arXiv:1501.00993] [INSPIRE].

[96] L. de Lima, C.S. Machado, R.D. Matheus and L.A.F. do Prado, Higgs Flavor Violation as a Signal to Discriminate Models, JHEP 11 (2015) 074 [arXiv:1501.06923] [INSPIRE].

[97] I. Doršner, S. Fajfer, A. Greljo, J.F. Kamenik, N. Košnik and I. Nišandžic, New Physics Models Facing Lepton Flavor Violating Higgs Decays at the Percent Level, JHEP 06 (2015) 108 [arXiv: 1502.07784] [INSPIRE].

[98] Y. Omura, E. Senaha and K. Tobe, Lepton-flavor-violating Higgs decay $h \rightarrow \mu \tau$ and muon anomalous magnetic moment in a general two Higgs doublet model, JHEP 05 (2015) 028 [arXiv: 1502.07824] [INSPIRE].

[99] D. Das and A. Kundu, Two hidden scalars around $125 \mathrm{GeV}$ and $h \rightarrow \mu \tau$, Phys. Rev. D 92 (2015) 015009 [arXiv: 1504.01125] [INSPIRE].

[100] Y.-n. Mao and S.-h. Zhu, On the Higgs- $\mu-\tau$ Coupling at High and Low Energy Colliders, arXiv:1505.07668 [INSPIRE].

[101] A. Crivellin, J. Heeck and P. Stoffer, A perturbed lepton-specific two-Higgs-doublet model facing experimental hints for physics beyond the Standard Model, arXiv:1507.07567 [INSPIRE].

[102] T. Goto, R. Kitano and S. Mori, Lepton flavor violating Z-boson couplings from nonstandard Higgs interactions, Phys. Rev. D 92 (2015) 075021 [arXiv:1507.03234] [INSPIRE].

[103] C.-W. Chiang, H. Fukuda, M. Takeuchi and T.T. Yanagida, Flavor-Changing Neutral-Current Decays in Top-Specific Variant Axion Model, JHEP 11 (2015) 057 [arXiv: 1507.04354] [INSPIRE].

[104] A. Crivellin, J. Heeck and P. Stoffer, A perturbed lepton-specific two-Higgs-doublet model facing experimental hints for physics beyond the Standard Model, arXiv:1507.07567 [INSPIRE].

[105] F.J. Botella, G.C. Branco, M. Nebot and M.N. Rebelo, Flavour Changing Higgs Couplings in a Class of Two Higgs Doublet Models, arXiv:1508.05101 [INSPIRE].

[106] Y. Omura, E. Senaha and K. Tobe, $\tau$ - and $\mu$-physics in a general two Higgs doublet model with $\mu-\tau$ flavor violation, arXiv:1511.08880 [INSPIRE].

[107] P. Minkowski, $\mu \rightarrow$ ey at a Rate of One Out of $10^{9}$ Muon Decays?, Phys. Lett. B 67 (1977) 421 [INSPIRE].

[108] T. Yanagida, Horizontal Symmetry And Masses Of Neutrinos, Conf. Proc. C 7902131 (1979) 95 [INSPIRE].

[109] M. Gell-Mann, P. Ramond and R. Slansky, Complex Spinors and Unified Theories, Conf. Proc. C 790927 (1979) 315 [arXiv: 1306.4669] [INSPIRE].

[110] S.L. Glashow, The Future of Elementary Particle Physics, NATO Sci. Ser. B 59 (1980) 687 [INSPIRE]. 
[111] R.N. Mohapatra and G. Senjanović, Neutrino Mass and Spontaneous Parity Violation, Phys. Rev. Lett. 44 (1980) 912 [INSPIRE].

[112] R.N. Mohapatra and G. Senjanović, Neutrino Masses and Mixings in Gauge Models with Spontaneous Parity Violation, Phys. Rev. D 23 (1981) 165 [INSPIRE].

[113] K.S. Babu and R.N. Mohapatra, Predictive neutrino spectrum in minimal $\mathrm{SO}(10)$ grand unification, Phys. Rev. Lett. 70 (1993) 2845 [hep-ph/9209215] [INSPIRE].

[114] K. Matsuda, Y. Koide and T. Fukuyama, Can the $\mathrm{SO}(10)$ model with two Higgs doublets reproduce the observed fermion masses?, Phys. Rev. D 64 (2001) 053015 [hep-ph/0010026] [INSPIRE].

[115] B. Bajc, A. Melfo, G. Senjanović and F. Vissani, Yukawa sector in non-supersymmetric renormalizable SO(10), Phys. Rev. D 73 (2006) 055001 [hep-ph/0510139] [INSPIRE].

[116] T. Fukuyama, K. Ichikawa and Y. Mimura, Revisiting fermion mass and mixing fits in the minimal SUSY SO(10) GUT, arXiv:1508.07078 [INSPIRE].

[117] Y. Zhang, H. An, X. Ji and R.N. Mohapatra, General CP-violation in Minimal Left-Right Symmetric Model and Constraints on the Right-Handed Scale, Nucl. Phys. B 802 (2008) 247 [arXiv:0712.4218] [INSPIRE].

[118] A. Maiezza, M. Nemevšek, F. Nesti and G. Senjanović, Left-Right Symmetry at LHC, Phys. Rev. D 82 (2010) 055022 [arXiv: 1005.5160] [InSPIRE].

[119] S. Bertolini, A. Maiezza and F. Nesti, Present and Future K and B Meson Mixing Constraints on TeV Scale Left-Right Symmetry, Phys. Rev. D 89 (2014) 095028 [arXiv:1403.7112] [INSPIRE].

[120] D. Gonçalves, F. Krauss and M. Spannowsky, Augmenting the diboson excess for the LHC Run II, Phys. Rev. D 92 (2015) 053010 [arXiv: 1508.04162] [InSPIRE].

[121] ATLAS collaboration, Identification of Boosted, Hadronically Decaying $W$ Bosons and Comparisons with ATLAS Data Taken at $\sqrt{s}=8 \mathrm{TeV}$, arXiv:1510.05821 [INSPIRE].

[122] CMS collaboration, Search for massive resonances in dijet systems containing jets tagged as $W$ or $Z$ boson decays in pp collisions at $\sqrt{s}=8$ TeV, JHEP 08 (2014) 173 [arXiv: 1405.1994] [INSPIRE].

[123] ATLAS collaboration, Search for $W W, W Z$ and $Z Z$ resonances in pp collisions at $\sqrt{s}=8 \mathrm{TeV}$ with the ATLAS detector, ATLAS-CONF-2015-045 (2015).

[124] ATLAS collaboration, Search for $W Z$ resonances in the fully leptonic channel using pp collisions at $\sqrt{s}=8 \mathrm{TeV}$ with the ATLAS detector, Phys. Lett. B 737 (2014) 223 [arXiv: 1406.4456] [INSPIRE].

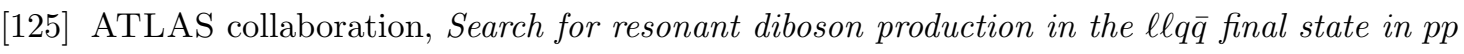
collisions at $\sqrt{s}=8 \mathrm{TeV}$ with the ATLAS detector, Eur. Phys. J. C 75 (2015) 69 [arXiv: 1409.6190] [INSPIRE].

[126] ATLAS collaboration, Search for production of $W W / W Z$ resonances decaying to a lepton, neutrino and jets in pp collisions at $\sqrt{s}=8 \mathrm{TeV}$ with the ATLAS detector, Eur. Phys. J. C 75 (2015) 209 [Erratum ibid. C 75 (2015) 370] [arXiv:1503.04677] [inSPIRE].

[127] CMS collaboration, Search for massive resonances decaying into pairs of boosted bosons in semi-leptonic final states at $\sqrt{s}=8 \mathrm{TeV}$, JHEP 08 (2014) 174 [arXiv:1405.3447] [INSPIRE]. 
[128] CMS collaboration, Search for new resonances decaying via WZ to leptons in proton-proton collisions at $\sqrt{s}=8 \mathrm{TeV}$, Phys. Lett. B $\mathbf{7 4 0}$ (2015) 83 [arXiv:1407.3476] [INSPIRE].

[129] CMS collaboration, Search for resonances and quantum black holes using dijet mass spectra in proton-proton collisions at $\sqrt{s}=8 \mathrm{TeV}$, Phys. Rev. D 91 (2015) 052009

[arXiv: 1501.04198] [INSPIRE].

[130] ATLAS collaboration, Search for new phenomena in the dijet mass distribution using $p-p$ collision data at $\sqrt{s}=8 \mathrm{TeV}$ with the ATLAS detector, Phys. Rev. D 91 (2015) 052007 [arXiv: 1407.1376] [INSPIRE].

[131] CMS collaboration, Search for massive WH resonances decaying to $\ell \nu \mathrm{b} \overline{\mathrm{b}}$ final state in the boosted regime at $\sqrt{s}=8 \mathrm{TeV}$, CMS-PAS-EXO-14-010.

[132] ATLAS collaboration, Search for a new resonance decaying to a $W$ or $Z$ boson and a Higgs boson in the $\ell \ell / \ell \nu / \nu \nu+b \bar{b}$ final states with the ATLAS detector, Eur. Phys. J. C 75 (2015) 263 [arXiv: 1503.08089] [INSPIRE].

[133] CMS collaboration, Search for A Massive Resonance Decaying into a Higgs Boson and a W or $Z$ Boson in Hadronic Final States in Proton-Proton Collisions at $\sqrt{s}=8 \mathrm{TeV}$, arXiv:1506.01443 [INSPIRE].

[134] ATLAS collaboration, Search for $W^{\prime} \rightarrow t b \rightarrow q q b b$ decays in pp collisions at $\sqrt{s}=8 \mathrm{TeV}$ with the ATLAS detector, Eur. Phys. J. C 75 (2015) 165 [arXiv:1408.0886] [INSPIRE].

[135] ATLAS collaboration, Search for $W^{\prime} \rightarrow t \bar{b}$ in the lepton plus jets final state in proton-proton collisions at a centre-of-mass energy of $\sqrt{s}=8 \mathrm{TeV}$ with the ATLAS detector, Phys. Lett. B 743 (2015) 235 [arXiv:1410.4103] [INSPIRE].

[136] CMS collaboration, Search for $W^{\prime} \rightarrow t b$ decays in the lepton + jets final state in $p p$ collisions at $\sqrt{s}=8 \mathrm{TeV}$, JHEP 05 (2014) 108 [arXiv:1402.2176] [INSPIRE].

[137] CMS collaboration, Search for $W^{\prime} \rightarrow$ tb in proton-proton collisions at $\sqrt{s}=8 \mathrm{TeV}$, arXiv: 1509.06051 [INSPIRE].

[138] J. Alwall et al., The automated computation of tree-level and next-to-leading order differential cross sections and their matching to parton shower simulations, JHEP 07 (2014) 079 [arXiv : 1405.0301] [INSPIRE].

[139] Q.-H. Cao, Z. Li, J.-H. Yu and C.P. Yuan, Discovery and Identification of W' and Z' in $\mathrm{SU}(2) \times \mathrm{SU}(2) \times \mathrm{U}(1)$ Models at the LHC, Phys. Rev. D 86 (2012) 095010 [arXiv: 1205.3769] [INSPIRE].

[140] T. Jezo, M. Klasen, D.R. Lamprea, F. Lyonnet and I. Schienbein, $N L O+N L L$ limits on $W^{\prime}$ and $Z^{\prime}$ gauge boson masses in general extensions of the Standard Model, JHEP 12 (2014) 092 [arXiv: 1410.4692] [INSPIRE].

[141] C. Grojean, E. Salvioni and R. Torre, A weakly constrained W' at the early LHC, JHEP 07 (2011) 002 [arXiv: 1103.2761] [inSPIRE].

[142] M.E. Peskin and T. Takeuchi, A New constraint on a strongly interacting Higgs sector, Phys. Rev. Lett. 65 (1990) 964 [INSPIRE].

[143] M.E. Peskin and T. Takeuchi, Estimation of oblique electroweak corrections, Phys. Rev. D 46 (1992) 381 [INSPIRE]. 
[144] K. Hsieh, K. Schmitz, J.-H. Yu and C.P. Yuan, Global Analysis of General $\mathrm{SU}(2) \times \mathrm{SU}(2) \times \mathrm{U}(1)$ Models with Precision Data, Phys. Rev. D 82 (2010) 035011 [arXiv: 1003.3482] [INSPIRE].

[145] F.F. Deppisch, T.E. Gonzalo, S. Patra, N. Sahu and U. Sarkar, Signal of Right-Handed Charged Gauge Bosons at the LHC?, Phys. Rev. D 90 (2014) 053014 [arXiv: 1407.5384] [INSPIRE].

[146] M. Heikinheimo, M. Raidal and C. Spethmann, Testing Right-Handed Currents at the LHC, Eur. Phys. J. C 74 (2014) 3107 [arXiv:1407.6908] [INSPIRE].

[147] F.F. Deppisch, T.E. Gonzalo, S. Patra, N. Sahu and U. Sarkar, Double beta decay, lepton flavor violation and collider signatures of left-right symmetric models with spontaneous D-parity breaking, Phys. Rev. D 91 (2015) 015018 [arXiv:1410.6427] [INSPIRE].

[148] ATLAS collaboration, Search for heavy Majorana neutrinos with the ATLAS detector in pp collisions at $\sqrt{s}=8 \mathrm{TeV}$, JHEP 07 (2015) 162 [arXiv: 1506.06020] [INSPIRE].

[149] R.N. Mohapatra, Mechanism for Understanding Small Neutrino Mass in Superstring Theories, Phys. Rev. Lett. 56 (1986) 561 [InSPIRE].

[150] R.N. Mohapatra and J.W.F. Valle, Neutrino Mass and Baryon Number Nonconservation in Superstring Models, Phys. Rev. D 34 (1986) 1642 [InSPIRE].

[151] S.M. Barr, A different seesaw formula for neutrino masses, Phys. Rev. Lett. 92 (2004) 101601 [hep-ph/0309152] [INSPIRE].

[152] M. Malinsky, J.C. Romao and J.W.F. Valle, Novel supersymmetric $\mathrm{SO}(10)$ seesaw mechanism, Phys. Rev. Lett. 95 (2005) 161801 [hep-ph/0506296] [INSPIRE].

[153] T. Fukuyama, T. Kikuchi and T. Osaka, Non-thermal leptogenesis and a prediction of inflaton mass in a supersymmetric $\mathrm{SO}(10)$ model, JCAP 06 (2005) 005 [hep-ph/0503201] [INSPIRE].

[154] S.P. Liew and S. Shirai, Testing ATLAS Diboson Excess with Dark Matter Searches at LHC, JHEP 11 (2015) 191 [arXiv:1507.08273] [INSPIRE].

[155] T. Cohen, M. Lisanti, A. Pierce and T.R. Slatyer, Wino Dark Matter Under Siege, JCAP 10 (2013) 061 [arXiv:1307.4082] [INSPIRE].

[156] J. Fan and M. Reece, In Wino Veritas? Indirect Searches Shed Light on Neutralino Dark Matter, JHEP 10 (2013) 124 [arXiv: 1307.4400] [INSPIRE].

[157] A. Hryczuk, I. Cholis, R. Iengo, M. Tavakoli and P. Ullio, Indirect Detection Analysis: Wino Dark Matter Case Study, JCAP 07 (2014) 031 [arXiv:1401.6212] [INSPIRE].

[158] B. Bhattacherjee, M. Ibe, K. Ichikawa, S. Matsumoto and K. Nishiyama, Wino Dark Matter and Future dSph Observations, JHEP 07 (2014) 080 [arXiv: 1405.4914] [INSPIRE].

[159] J. Hisano, K. Ishiwata and N. Nagata, QCD Effects on Direct Detection of Wino Dark Matter, JHEP 06 (2015) 097 [arXiv: 1504.00915] [INSPIRE].

[160] CMS collaboration, Search for narrow resonances decaying to dijets in proton-proton collisions at $\sqrt{s}=13 \mathrm{TeV}$, arXiv:1512.01224 [INSPIRE].

[161] ATLAS collaboration, Search for new phenomena in dijet mass and angular distributions from pp collisions at $\sqrt{s}=13 \mathrm{TeV}$ with the ATLAS detector, Phys. Lett. B 754 (2016) 302 [arXiv: 1512.01530] [INSPIRE]. 